\title{
Variational inequalities for the commutators of rough operators with BMO functions *
}

\author{
Yanping Chen 1 \\ Department of Applied Mathematics, School of Mathematics and Physics, \\ University of Science and Technology Beijing, \\ Beijing 100083, The People's Republic of China \\ E-mail: yanpingch@126.com \\ Yong Ding \\ School of Mathematical Sciences, Beijing Normal University, \\ Laboratory of Mathematics and Complex Systems (BNU), Ministry of Education, \\ Beijing 100875, The People's Republic of China \\ E-mail: dingy@bnu.edu.cn \\ Guixiang Hong \\ School of Mathematics and Statistics, Wuhan University, \\ Wuhan 430072, The People's Republic of China \\ E-mail: guixiang.hong@whu.edu.cn \\ and \\ Honghai Liu \\ School of Mathematics and Information Science, \\ Henan Polytechnic University, Jiaozuo, Henan, 454003, China. \\ E-mail: hhliu@hpu.edu.cn
}

MR(2000) Subject Classification: 42B $20,42 \mathrm{~B} 25$

Keywords: Commutators; variational inequalities; singular integrals; averaging operators; rough kernels

${ }^{*}$ The research was supported by NSF of China (Grant: 11471033, 11371057, 11571160, 11601396), Thousand Youth Talents Plan of China (Grant: 429900018-101150(2016)), Funds for Talents of China (Grant: 413100002), the Fundamental Research Funds for the Central Universities (FRF-BR-16-011A, 2014KJJCA10) and SRFDP of China (Grant: 20130003110003).

${ }^{1}$ Corresponding author. 


\begin{abstract}
In this paper, starting with a relatively simple observation that the variational estimates of the commutators of the standard Calderón-Zygmund operators with the BMO functions can be deduced from the weighted variational estimates of the standard Calderón-Zygmund operators themselves, we establish similar variational estimates for the commutators of the BMO functions with rough singular integrals which do not admit any weighted variational estimates. The proof involves many LittlewoodPaley type inequalities with commutators as well as Bony decomposition and related para-product estimates.
\end{abstract}

\title{
1 Introduction
}

Motivated by the modulus of continuity of Brownian motion, Lépingle [39] established the first variational inequality for general martingales (see [48] for a simple proof). Bourgain [4] is the first one who exploited Lépingle's result to obtain corresponding variational estimates for the Birkhoff ergodic averages along subsequences of natural numbers and then directly deduce pointwise convergence results without previous knowledge that pointwise convergence holds for a dense subclass of functions, which are not available in some ergodic models. In particular, Bourgain's work [4] has initiated a new research direction in ergodic theory and harmonic analysis. In [32, [34], 33], 5], 6], Jones and his collaborators systematically studied variational inequalities for ergodic averages and truncated singular integrals of homogeneous type. Since then many other publications came to enrich the literature on this subject (cf. e.g. [23, [38], 20], [35], [43, [47], [26]). Recently, several works on weighted as well as vector-valued variational inequalities in ergodic theory and harmonic analysis have also appeared (cf. e.g. [41], 42, [37, [31, [27], [28]); and several results on $\ell^{p}\left(\mathbb{Z}^{d}\right)$-estimates of $q$-variations for discrete operators of Radon type have also been established (cf. e.g. [36], 44], [45], 46], 55]).

Most of the operators considered in the previous cited papers are of homogeneous type, and it is still unknown whether variational inequalities hold for all singular integrals of convolution type (while it is true when the kernel is smooth enough in [45]), let alone for all standard Calderón-Zygmund operators. In our another paper [11, we consider the variational inequality for Calderón commutators - commutators of singular integrals or pseudo differential operators with Lipschitz functions, which are typical example of Calderón-Zygmund operators of nonconvolution type. In the present paper, we establish variational estimates for the commutators of rough operators with BMO functions, which finds its motivation in a simple observation that that the variational estimates of the commutators of the standard Calderón-Zygmund operators with the BMO functions can be deduced from the weighted variational estimates of the standard Calderón-Zygmund operators themselves.

To illustrate the observation, let us introduce some notations and recall some notions. Given a family of complex numbers $\mathfrak{a}=\left\{a_{t}: t \in \mathbb{R}\right\}$ and $\rho \geq 1$, the $\rho$-variation norm of the family $\mathfrak{a}$ is defined by

$$
\|\mathfrak{a}\|_{V_{\rho}}=\sup \left(\sum_{k \geq 1}\left|a_{t_{k}}-a_{t_{k-1}}\right|^{\rho}\right)^{\frac{1}{\rho}}
$$


where the supremum runs over all increasing sequences $\left\{t_{k}: k \geq 0\right\}$. It is trivial that

$$
\|\mathfrak{a}\|_{L^{\infty}(\mathbb{R})}:=\sup _{t \in \mathbb{R}}\left|a_{t}\right| \leq\|\mathfrak{a}\|_{V_{\rho}}+\left|a_{t_{0}}\right| \quad \text { for } \rho \geq 1
$$

for some fixed $t_{0}$.

Let $0<\rho<\infty$. Given a family of Lebesgue measurable functions $\mathcal{F}=\left\{F_{t}: t \in \mathbb{R}_{+}\right\}$defined on $\mathbb{R}^{n}$, we define the strong $\rho$-variation function $V_{\rho}(\mathcal{F})$ of the family $\mathcal{F}$ as

$$
V_{\rho}(\mathcal{F})(x)=\sup \left\|\left(F_{t_{k}}(x)-F_{t_{k-1}}(x)\right)_{k \geq 1}\right\|_{\ell^{\rho}}, \text { a.e. } x \in \mathbb{R}^{n}
$$

where the supremum runs over all increasing sequences $\left\{t_{k}: k \geq 0\right\}$. Suppose $\mathcal{T}=\left\{T_{t}\right\}_{t>0}$ is a family of operators on $L^{p}\left(\mathbb{R}^{n}\right)(1 \leq p \leq \infty)$. The strong $\rho$-variation operator is simply defined as

$$
V_{\rho} \mathcal{T}(f)(x)=\left\|\left\{T_{t}(f)(x)\right\}_{t>0}\right\|_{V_{\rho}}, \quad \forall f \in L^{p}\left(\mathbb{R}^{n}\right) .
$$

Thus the operator $V_{\rho} \mathcal{T}$ sends functions on $\mathbb{R}^{n}$ to nonnegative functions on $\mathbb{R}^{n}$. It is easy to observe from the definition of $\rho$-variation norm that for any $x$ if $V_{\rho} \mathcal{T}(f)(x)<\infty$, then $\left\{T_{t}(f)(x)\right\}_{t>0}$ converges when $t \rightarrow 0$ or $t \rightarrow \infty$. In particular, if $V_{\rho} \mathcal{T}(f)$ belongs to some function spaces such as $L^{p}\left(\mathbb{R}^{n}\right)$ or $L^{p, \infty}\left(\mathbb{R}^{n}\right)$, then the sequence converges almost everywhere without any additional condition. This is why mapping property of strong $\rho$-variation operator is so interesting in ergodic theory and harmonic analysis. Also, by (1.2), for any $f \in L^{p}\left(\mathbb{R}^{n}\right)$ and $x \in \mathbb{R}^{n}$, we have

$$
T^{*}(f)(x) \leq V_{\rho}(\mathcal{T} f)(x) \text { for } \rho \geq 1 \text {, }
$$

where $T^{*}$ is the maximal operator defined by

$$
T^{*}(f)(x):=\sup _{t>0}\left|T_{t}(f)(x)\right|
$$

For $b \in B M O\left(\mathbb{R}^{n}\right)$ and $u \in \mathbb{N}$. If $T$ is a linear operator on some measurable function space, then the $u$-th order commutator formed by $b$ and $T$ is defined by $T_{b, u} f(x):=T((b(x)-$ $\left.b(\cdot))^{u} f\right)(x)$. Simply, define $T_{b}:=T_{b, 1}$. In 1976, Coifman, Rochberg and Weiss 17 obtained a characterization of $L^{p}$-boundedness of the commutators $R_{j ; b}$ generated by the Reisz transforms $R_{j}(j=1, \cdots, n$,$) and a BMO function b$. As an application of this characterization, a decomposition theorem of the real Hardy space is given in this paper. Moreover, the authors in [17] proved also that if $\Omega \in \operatorname{Lip}\left(S^{n-1}\right)$, then the commutator $T_{\Omega ; b}$ for $T_{\Omega}$ and a BMO function $b$ is bounded on $L^{p}$ for $1<p<\infty$, which is defined by

$$
T_{\Omega ; b} f(x)=\text { p.v. } \int_{\mathbb{R}^{n}} \frac{\Omega(x-y)}{|x-y|^{n}}(b(x)-b(y)) f(y) d y .
$$

In the same paper, Coifman, Rochberg and Weiss [17 outlined a different approach, which is less direct but shows the close relationship between the weighted inequalities of the operator $T$ and the weighted inequalities of the commutator $T_{b}$. In 1993, Alvarez, Bagby, Kurtz and Pérez 11 developed the idea of 17, and established a generalized boundedness criterion for the commutators of linear operators. As it is well-known that the commutators have played an important role in harmonic analysis and PDE, for example in the theory of non-divergent elliptic 
equations with discontinuous coefficients (see [8], 2], [9], [13, [14, [19, [51]). Moreover, there is also an interesting connection between the nonlinear commutator, considered by Rochberg and Weiss in 50, and Jacobian mapping of vector functions. They have been applied in the study of the nonlinear partial differential equations (see [16, 25]).

In order to state our main results, let us first recall the definition and some properties of $A_{p}$ weight on $\mathbb{R}$. Let $w$ be a non-negative locally integrable function defined on $\mathbb{R}^{n}$. We say $w \in A_{1}$ if there is a constant $C>0$ such that $M(w)(x) \leq C w(x)$, where $M$ is the classical Hardy-Littlewood maximal operator defined by

$$
M f(x)=\sup _{r>0} \frac{1}{r^{n}} \int_{|y| \leq r}|f(x-y)| d y .
$$

Equivalently, $w \in A_{1}$ if and only if there is a constant $C>0$ such that for any cube $Q$

$$
\frac{1}{|Q|} \int_{Q} w(x) d x \leq C \inf _{x \in Q} w(x)
$$

For $1<p<\infty$, we say that $w \in A_{p}$ if there exists a constant $C>0$ such that

$$
\sup _{Q}\left(\frac{1}{|Q|} \int_{Q} w(x) d x\right)\left(\frac{1}{|Q|} \int_{Q} w(x)^{1-p^{\prime}} d x\right)^{p-1} \leq C .
$$

The smallest constant appearing in (1.4) or (1.5) is denoted by $[w]_{A_{p}}$.

Now we state our first result as follows.

Theorem 1.1. Let $b \in B M O\left(\mathbb{R}^{n}\right)$. Let $\mathcal{T}=\left\{T_{t}: t \in \mathbb{R}_{+}\right\}$be a family of linear operators and $\mathcal{T}_{b}=\left\{T_{t ; b}: t \in \mathbb{R}_{+}\right\}$be the family of commutators formed by the linear operators and $b$. Let $1<p<\infty, \rho \geq 1, \tau \in \mathbb{R}$ and $1<s<\infty$. Let $w$ be a locally integrable function such that $w^{\tau} \in A_{s}$. Then $V_{\rho} \mathcal{T}_{b}$ is bounded on $L^{p}(w)$, that is,

$$
\left\|V_{\rho} \mathcal{T}_{b}(f)\right\|_{L^{p}(w)} \leq C\|b\|_{*}\|f\|_{L^{p}(w)}
$$

provided that $V_{\rho} \mathcal{T}$ is bounded on $L^{p}(w)$.

Now we present four consequences of Theorem 1.1. Let $K$ be a kernel on $\mathbb{R}^{n} \times \mathbb{R}^{n} \backslash\{(x, x)\}$ : $\left.x \in \mathbb{R}^{n}\right\}$. We will suppose that $K$ satisify the following regularity conditions. There exist two constants $\delta>0$ and $C>0$ such that

$$
\begin{gathered}
|K(x, y)| \leq \frac{C}{|x-y|^{n}}, \text { for } x \neq y ; \\
|K(x, y)-K(z, y)| \leq \frac{C|x-z|^{\delta}}{|x-y|^{n+\delta}}, \text { for }|x-y|>2|x-z| ; \\
|K(y, x)-K(y, z)| \leq \frac{C|x-z|^{\delta}}{|x-y|^{n+\delta}}, \text { for }|x-y|>2|x-z| .
\end{gathered}
$$


Let $T$ be the standard Calderón-Zygmund operator associated to the kernel $K$ : For a Schwartz function $f$,

$$
T f(x):=\lim _{\varepsilon \rightarrow 0^{+}} T_{\varepsilon} f
$$

where $T_{\varepsilon}$ be the truncated operator

$$
T_{\varepsilon} f(x)=\int_{|x-y|>\varepsilon} K(x, y) f(y) d y
$$

for $b \in B M O\left(\mathbb{R}^{n}\right)$, define $T_{\varepsilon ; b}$ to be the truncated commutator

$$
T_{\varepsilon ; b} f(x)=\int_{|x-y|>\varepsilon} K(x, y)(b(x)-b(y)) f(y) d y
$$

Denote by $\mathcal{T}=\left\{T_{\varepsilon}\right\}_{\varepsilon>0}$ and $\mathcal{T}_{b}=\left\{T_{\varepsilon ; b}\right\}_{\varepsilon>0}$. Let $K$ be kernel on $\mathbb{R}^{n}$ satisfying (1.6)-(1.8), and let $2<\rho<\infty$. In [42], they showed that if the operator $V_{\rho} \mathcal{T}$ is of type $\left(p_{0}, p_{0}\right)$ for some $1<p_{0}<\infty$, then for $1<p<\infty$ and $w \in A_{p}, V_{\rho} \mathcal{T}$ is bounded on $L^{p}(w)$. Thus apply Theorem 1.1, we get

Corollary 1.2. Let $b \in B M O\left(\mathbb{R}^{n}\right)$ and $K$ be kernel on $\mathbb{R}^{n}$ satisfying (1.6)-(1.8). Let $2<\rho<\infty$. If $V_{\rho} \mathcal{T}$ is of type $\left(p_{0}, p_{0}\right)$ for some $1<p_{0}<\infty$, then for $1<p<\infty$ and $w \in A_{p}$, there exists a constant $C$ such that

$$
\left\|V_{\rho} \mathcal{T}_{b}(f)\right\|_{L^{p}(w)} \leq C\|b\|_{*}\|f\|_{L^{p}(w)} .
$$

In particular, if $K$ is of convolution type satisfying cancellation condition

$$
\int_{\partial B(0, t)} K(x) d x=0, \forall t>0
$$

and either (i) $|\nabla K(x)| \leq C \frac{1}{|x|^{n+1}}$ or (ii) $K(x)=\frac{\Omega(x)}{|x|^{n}}$ with $\Omega \in \operatorname{Lip}_{\delta}\left(\mathbf{S}^{n}\right)$, then the associated $V_{\rho} \mathcal{T}_{b}$ if of type $L^{p}(w)$.

We refer the reader to [45] (resp. [6]) for the result $V_{\rho} \mathcal{T}$ is $L^{2}$-bounded when $K$ satisfies (i) (resp. (ii)). On the other hand, Corollary 1.2 implies the main result of [40] where $T$ is the Hilbert transform.

Let $\varphi: \mathbb{R}^{n} \rightarrow[0,+\infty)$ be a radially decreasing integrable function. Let $\varphi_{t}(x)=\frac{1}{t^{n}} \varphi\left(\frac{x}{t}\right)$ and $\Phi(f)(x)=\left\{\varphi_{t} * f(x)\right\}_{t>0}$. In [42], they also showed that for $2<\rho<\infty$, the operator $V_{\rho} \Phi$ is bounded on $L^{p}(w)$ for $1<p<\infty$ and $w \in A_{p}$. Denote by $\Phi_{b}(f)=\left\{\varphi_{t}((b(x)-b(\cdot)) f)\right\}_{t>0}$. Thus apply Theorem 1.1, we have

Corollary 1.3. Let $b \in B M O\left(\mathbb{R}^{n}\right)$. Let $\varphi: \mathbb{R}^{n} \rightarrow[0,+\infty)$ be a radially decreasing integrable function. Let $\varphi_{t}(x)=\frac{1}{t^{n}} \varphi\left(\frac{x}{t}\right)$ and $\Phi_{b}(f)(x)=\left\{\varphi_{t}((b(x)-b(\cdot)) f)(x)\right\}_{t>0}$. For $2<\rho<\infty$, $1<p<\infty$ and $w \in A_{p}$, there exists a constant $C$ such that

$$
\left\|V_{\rho} \Phi_{b}(f)\right\|_{L^{p}(w)} \leq C\|b\|_{*}\|f\|_{L^{p}(w)} .
$$


Not only to smooth singular kernels or good approximation identities, Theorem 1.1 can also be applied to singular integral operators or averaging operators with some homogeneous rough kernels. Suppose $T_{\Omega, \varepsilon}$ is the truncated singular integral operator defined by

$$
T_{\Omega, \varepsilon} f(x)=\int_{|y|>\varepsilon} \frac{\Omega\left(y^{\prime}\right)}{|y|^{n}} f(x-y) d y
$$

where $\Omega \in L^{1}\left(\mathbf{S}^{n-1}\right)$ satisfies the cancelation condition

$$
\int_{\mathbf{S}^{n-1}} \Omega\left(y^{\prime}\right) d \sigma\left(y^{\prime}\right)=0 .
$$

For $1<p<\infty$ and $f \in C_{c}^{\infty}\left(\mathbb{R}^{n}\right)$, the Calderón-Zygmund singular integral operator $T$ with homogeneous kernel is defined by

$$
T_{\Omega} f(x)=\lim _{\varepsilon \rightarrow 0^{+}} T_{\varepsilon} f(x) \text {, a.e. } x \in \mathbb{R}^{n} .
$$

Denote the family of operators $\left\{T_{\Omega, \varepsilon}\right\}_{\varepsilon>0}$ by $\mathcal{T}_{\Omega}$. In [10], we showed that if $\Omega \in L^{q}\left(\mathbf{S}^{n-1}\right), q>1$ satisfying (1.11), then for $\rho>2, V_{\rho} \mathcal{T}_{\Omega}$ is bounded on $L^{p}(w)$, whenever $w$ and $p$ satisfy one of the following conditions:

(i) $q^{\prime} \leq p<\infty, p \neq 1$ and $w \in A_{p / q^{\prime}}$,

(ii) $1<p \leq q, p \neq \infty$ and $w^{-\frac{1}{(p-1)}} \in A_{p^{\prime} / q^{\prime}}$.

Thus, we can invoke Theorem [1.1 to obtain the corresponding weighted estimates for the strong $\rho$ variation of commutators with rough kernel and $b \in B M O\left(\mathbb{R}^{n}\right)$.

Corollary 1.4. Let $b \in B M O\left(\mathbb{R}^{n}\right)$ and $\mathcal{T}_{\Omega ; b} f(x)$ be the family of commutators $\left\{T_{\Omega, \varepsilon}((b(x)-\right.$ $b(\cdot) f)(x))\}_{\varepsilon>0}$ with $\Omega \in L^{q}\left(\mathbf{S}^{n-1}\right), q>1$ satisfying (1.11), then for $\rho>2$, there exists a constant $C$ such that

$$
\left\|V_{\rho} \mathcal{T}_{b}(f)\right\|_{L^{p}(w)} \leq C\|b\|_{*}\|f\|_{L^{p}(w)},
$$

if $w$ and $p$ satisfy one of the situation (i) or (ii).

We can also give applications of Theorem 1.1 to the situation of averaging operators with rough kernels $\mathcal{M}_{\Omega}=\left\{M_{\Omega, t}\right\}_{t>0}$, where $M_{\Omega, t}$ is defined as

$$
M_{\Omega, t} f(x)=\frac{1}{t^{n}} \int_{|y|<t} \Omega\left(y^{\prime}\right) f(x-y) d y,
$$

where $\Omega \in L^{1}\left(\mathbf{S}^{n-1}\right)$. In [10], we showed that if $\Omega \in L^{q}\left(\mathbf{S}^{n-1}\right), q>1$, then for $\rho>2, V_{\rho} \mathcal{M}_{\Omega}$ is bounded on $L^{p}(w)$ if $w$ and $p$ satisfy one of the conditions (i) and (ii). See [22, 9] on the maximal inequality for the family $\mathcal{M}_{\Omega}$. Given a BMO function $b$, denote the family of operators $\left\{M_{\Omega, t}(b(x)-b(\cdot) f)(x)\right\}_{t>0}$ by $\mathcal{M}_{\Omega ; b} f(x)$. Thus, we can invoke Theorem 1.1 to obtain the corresponding weighted estimates for the strong $\rho$ variation of commutators with rough kernel and $b \in B M O\left(\mathbb{R}^{n}\right)$. 
Corollary 1.5. Let $b \in B M O\left(\mathbb{R}^{n}\right)$ and $\mathcal{M}_{\Omega ; b}$ be the family of the commutators of averaging operators with $\Omega \in L^{q}\left(\mathbf{S}^{n-1}\right), q>1$. Then for $\rho>2$, there exists a constant $C$ such that

$$
\left\|V_{\rho} \mathcal{M}_{\Omega ; b}(f)\right\|_{L^{p}(w)} \leq C\|b\|_{*}\|f\|_{L^{p}(w)}
$$

if $w$ and $p$ satisfy one of the conditions (i) or (ii).

However, it is not clear up to now whether the operator $V_{\rho} \mathcal{T}_{\Omega}$ or $V_{\rho} \mathcal{M}_{\Omega}$ with $\Omega \in L^{1} \backslash$ $\bigcup_{q>1} L^{q}\left(S^{n-1}\right)$ is bounded on $L^{p}(w)(1<p<\infty)$ for all $w \in A_{r}(1<r<\infty)$, Hence, if $\Omega \in L^{1} \backslash \bigcup_{q>1} L^{q}\left(S^{n-1}\right)$, the $L^{p}$ boundedness of $V_{\rho} \mathcal{T}_{\Omega ; b}$ and $V_{\rho} \mathcal{M}_{\Omega ; b}$ can not be deduced from Theorem [1.1.

The main purpose of this paper is to give a sufficient condition which contains $\bigcup_{q>1} L^{q}\left(S^{n-1}\right)$, such that the operators $V_{\rho} \mathcal{T}_{\Omega, b}$ and $V_{\rho} \mathcal{M}_{\Omega ; b}$ are bounded on $L^{p}\left(\mathbb{R}^{n}\right)$ for $1<p<\infty$. It is well known that

$$
\bigcup_{q>1} L^{q}\left(S^{n-1}\right) \subset L\left(\log ^{+} L\right)^{\alpha}\left(S^{n-1}\right)
$$

for any $\alpha>0$.

The second main result of this paper is formulated as follows.

Theorem 1.6. Let $b \in B M O\left(\mathbb{R}^{n}\right)$ and $\mathcal{T}_{\Omega ; b}$ be the family of the commutators of truncated singular integral operators with $\Omega$ satisfying (1.11). If $\Omega \in L\left(\log ^{+} L\right)^{3}\left(\mathbf{S}^{n-1}\right)$, then the following $\rho(2<\rho<\infty)$-variational inequality holds for $1<p<\infty$, namely,

$$
\left\|V_{\rho} \mathcal{T}_{\Omega ; b}(f)\right\|_{L^{p}} \leq C\|b\|_{*}\|f\|_{L^{p}}
$$

The proof of this result is based on Fourier transform, which is somehow standard but technical since it involves many Littlewood-Paley type inequalities with commutators as well as Bony decomposition and related para-product estimates.

Our approach to the variational estimates for singular integrals also works for the family $\mathcal{M}_{\Omega ; b}$.

Theorem 1.7. Let $1<p<\infty$ and Let $b \in B M O\left(\mathbb{R}^{n}\right)$. If $\Omega \in L\left(\log ^{+} L\right)^{2}\left(\mathbf{S}^{n-1}\right)$, then the $\rho(2<\rho<\infty)$-variational inequality for the family $\mathcal{M}_{\Omega ; b}$ holds,

$$
\left\|V_{\rho} \mathcal{M}_{\Omega ; b}(f)\right\|_{L^{p}} \leq C\|b\|_{*}\|f\|_{L^{p}}, \forall f \in L^{p}\left(\mathbb{R}^{n}\right) .
$$

The paper is organized as follows. In Section 2, we give the proof of Theorem 1.1, In Section 3 , we give the proof of Theorem 1.6. Section 4 is devoted to the proof of Theorem 1.7. For $p \geq 1$, $p^{\prime}$ denotes the conjugate exponent of $p$, that is, $p^{\prime}=p /(p-1)$. Throughout this paper, the letter " $C$ " will stand for a positive constant which is independent of the essential variables and not necessarily the same one in each occurrence. 


\section{Proof of Theorem 1.1}

Denote $F(z)=e^{z[b(x)-b(y)]}, z \in \mathbb{C}$. Then by the analyticity of $F(z)$ on $\mathbb{C}$ and the Cauchy integration formula, we have for any $\varepsilon>0$

$$
\begin{aligned}
b(x)-b(y)=F^{\prime}(0) & =\frac{1}{2 \pi i} \int_{|z|=\varepsilon} \frac{F(z)}{z^{2}} d z \\
& =\frac{1}{2 \pi} \int_{0}^{2 \pi} \frac{F\left(\varepsilon e^{i \theta}\right)}{\varepsilon e^{i \theta}} d \theta \\
& =\frac{1}{2 \pi \varepsilon} \int_{0}^{2 \pi} e^{\varepsilon e^{i \theta}[b(x)-b(y)]} e^{-i \theta} d \theta .
\end{aligned}
$$

For any linear operator $T$ we get

$$
\begin{aligned}
T_{b} f(x)=T(b(x)-b(\cdot) f)(x) & =\frac{1}{2 \pi \varepsilon} \int_{0}^{2 \pi} T\left(e^{-b(\cdot) \varepsilon e^{i \theta}} f\right)(x) e^{b(x) \varepsilon e^{i \theta}} e^{-i \theta} d \theta \\
& =\frac{1}{2 \pi \varepsilon} \int_{0}^{2 \pi} T\left(h_{\theta}\right)(x) e^{b(x) \varepsilon e^{i \theta}} e^{-i \theta} d \theta
\end{aligned}
$$

where $h_{\theta}(x)=f(x) e^{-b(x) \varepsilon e^{i \theta}}$ for $\theta \in[0,2 \pi]$. Recall that

$$
V_{\rho}(\mathcal{F})(x)=\sup \left\|\left(F_{t_{k}}(x)-F_{t_{k-1}}(x)\right)_{k \geq 1}\right\|_{\ell^{\rho}},
$$

where the supremum runs over all increasing sequences $\left\{t_{k}: k \geq 0\right\}$. Then using the Minkowski inequality, we have for $w^{\tau} \in A_{s}$

$$
\begin{aligned}
& \left\|V_{\rho} \mathcal{T}_{b}(f)\right\|_{L^{p}(w)} \\
& =\|\sup \|\left(T_{t_{k} ; b} f-T_{t_{k-1} ; b} f\right)_{k \geq 1}\left\|_{\ell^{\rho}}\right\|_{L^{p}(w)} \\
& =\left(\left.\int_{\mathbb{R}^{n}}\left|\sup \left\|\left(\frac{1}{2 \pi \varepsilon} \int_{0}^{2 \pi}\left(T_{t_{k}}\left(h_{\theta}\right)(x)-T_{t_{k-1}}\left(h_{\theta}\right)(x)\right) e^{b(x) \varepsilon e^{i \theta}} e^{-i \theta} d \theta\right)_{k \geq 1}\right\|_{\ell^{\rho}}\right|\right|^{p} w(x) d x\right)^{1 / p} \\
& \leq \frac{1}{2 \pi \varepsilon} \int_{0}^{2 \pi}\left(\int_{\mathbb{R}^{n}}\left|\sup \left\|\left(T_{t_{k}}\left(h_{\theta}\right)(x)-T_{t_{k-1}}\left(h_{\theta}\right)(x)\right)_{k \geq 1}\right\| \ell_{\ell^{\rho}}\right|^{p} e^{p b(x) \varepsilon \cos \theta} w(x) d x\right)^{1 / p} d \theta \\
& =\frac{1}{2 \pi \varepsilon} \int_{0}^{2 \pi}\left\|V_{\rho} \mathcal{T}\left(h_{\theta}\right)\right\|_{L^{p}\left(w e^{p b \varepsilon \cos \theta}\right)} d \theta .
\end{aligned}
$$

Note that for $f \in L^{p}(w)$, it is easy to check that for any $\theta \in[0,2 \pi]$,

$$
h_{\theta} \in L^{p}\left(w e^{p b(\cdot) \varepsilon \cos \theta}\right) \text { and }\left\|h_{\theta}\right\|_{L^{p}\left(w e^{p b(\cdot) \varepsilon \cos \theta}\right)}=\|f\|_{L^{p}(w)} .
$$

Hence we should compute

$$
\left[e^{\tau p b \varepsilon \cos \theta} w^{\tau}\right]_{A_{s}}=\sup _{Q}\left(\frac{1}{|Q|} \int_{Q} e^{\tau p b(x) \varepsilon \cos \theta} w^{\tau}(x) d x\right)\left(\frac{1}{|Q|} \int_{Q}\left[e^{\tau p b(x) \varepsilon \cos \theta} w^{\tau}(x)\right]^{-\frac{1}{s-1}} d x\right)^{s-1} .
$$


Now, since $w^{\tau} \in A_{s}$, there exists some $r_{1}>1$ such that

$$
\left(\frac{1}{|Q|} \int_{Q} w^{\tau r_{1}} d x\right)^{\frac{1}{r_{1}}} \leq \frac{2}{|Q|} \int_{Q} w^{\tau} d x
$$

and similarly for $w^{-\frac{\tau}{s-1}} \in A_{s^{\prime}}$, there exists some $r_{2}>1$ such that then

$$
\left(\frac{1}{|Q|} \int_{Q} w^{-\frac{\tau r_{2}}{s-1}} d x\right)^{\frac{1}{r_{2}}} \leq \frac{2}{|Q|} \int_{Q} w^{-\tau /(s-1)} d x .
$$

By this, we know if $r_{2}<r_{1}$, by the Hölder inequality, we get

$$
\left(\frac{1}{|Q|} \int_{Q} w^{\tau r_{2}} d x\right)^{\frac{1}{r_{2}}} \leq\left(\frac{1}{|Q|} \int_{Q} w^{\tau r_{1}} d x\right)^{\frac{1}{r_{1}}} \leq \frac{2}{|Q|} \int_{Q} w^{\tau} d x .
$$

If $r_{1}<r_{2}$, by the Hölder inequality, we get

$$
\left(\frac{1}{|Q|} \int_{Q} w^{-\frac{\tau r_{1}}{s-1}} d x\right)^{\frac{1}{r_{1}}} \leq\left(\frac{1}{|Q|} \int_{Q} w^{-\frac{\tau r_{2}}{s-1}} d x\right)^{\frac{1}{r_{2}}} \leq \frac{2}{|Q|} \int_{Q} w^{-\frac{\tau}{s-1}} d x .
$$

Taking $r=\min \left\{r_{1}, r_{2}\right\}$, using (2.3), (2.4) and the Hölder inequality we have

$$
\begin{aligned}
& {\left[e^{\tau p b \varepsilon \cos \theta} w^{\tau}\right]_{A_{s}} } \\
= & \sup _{Q}\left(\frac{1}{|Q|} \int_{Q} e^{\tau p b(x) \varepsilon \cos \theta} w^{\tau}(x) d x\right)\left(\frac{1}{|Q|} \int_{Q} e^{-\tau \frac{s}{s-1} b(x) \varepsilon \cos \theta} w(x)^{-\frac{\tau}{s-1}} d x\right)^{s-1} \\
\leq & \sup _{Q}\left(\frac{1}{|Q|} \int_{Q} w^{\tau r}(x) d x\right)^{\frac{1}{r}}\left(\frac{1}{|Q|} \int_{Q} e^{\tau p r^{\prime} b(x) \varepsilon \cos \theta} d x\right)^{\frac{1}{r^{\prime}}} \\
& \left(\frac{1}{|Q|} \int_{Q} w(x)^{-\tau r /(s-1)} d x\right)^{\frac{s-1}{r}}\left(\frac{1}{|Q|} \int_{Q} e^{-\frac{\tau p}{s-1} r^{\prime} b(x) \varepsilon \cos \theta} d x\right)^{\frac{s-1}{r^{\prime}}} \\
\leq & 4 \sup _{Q}\left(\frac{1}{|Q|} \int_{Q} w^{\tau}(x) d x\right)\left(\frac{1}{|Q|} \int_{Q} w(x)^{-\tau /(s-1)} d x\right)^{s-1} \\
& \sup _{Q}\left(\frac{1}{|Q|} \int_{Q} e^{\tau p r^{\prime} b(x) \varepsilon \cos \theta} d x\right)^{\frac{1}{r^{\prime}}}\left(\frac{1}{|Q|} \int_{Q} e^{-\frac{\tau p}{s-1} r^{\prime} b(x) \varepsilon \cos \theta} d x\right)^{\frac{s-1}{r^{\prime}}} \\
= & 4\left[w^{\tau}\right]_{A_{s}}\left[e^{\tau p r^{\prime} b \varepsilon \cos \theta}\right]_{A_{s}}^{\frac{1}{r^{\prime}}} .
\end{aligned}
$$

Now, since $b \in B M O\left(\mathbb{R}^{n}\right)$ we apply Lemma 2.1 to do this.

Lemma 2.1. ([15]) Let $1<p<\infty$ and $b \in B M O$. There exist two dimensional constants $\alpha_{n}, \beta_{n}$ satisfying $0<\alpha_{n}<1$ and $0<\beta_{n}<\infty$ such that for any $\lambda \in \mathbb{R}$ with $|\lambda| \leq \frac{\alpha_{n}}{\|b\|_{*}} \min \left\{1, \frac{1}{p-1}\right\}$, we have

$$
e^{\lambda b} \in A_{p} \text { and }\left[e^{\lambda b}\right]_{A_{p}} \leq \beta_{n}^{p}
$$


Now, we choose the radius

$$
\varepsilon=\frac{\alpha_{n} \min \left\{1, \frac{s^{\prime}}{s}\right\}}{\tau p r^{\prime}\|b\|_{*}}
$$

such that $\left|\tau p r^{\prime} \varepsilon \cos \theta\right| \leq \frac{\alpha_{n}}{\|b\|_{*}} \min \left\{1, \frac{s^{\prime}}{s}\right\}$, then by Lemma 2.1, we get

$$
\left[e^{\tau p r^{\prime} b \varepsilon \cos \theta}\right]_{A_{s}} \leq \beta_{n}^{s} .
$$

Combining (2.5) and (2.7), we get

$$
\left[e^{\tau p b \varepsilon \cos \theta} w^{\tau}\right]_{A_{s}} \leq 4\left[w^{\tau}\right]_{A_{s}}\left[e^{\tau p r^{\prime} b \varepsilon \cos \theta}\right]_{A_{s}}^{\frac{1}{r^{\prime}}} \leq 4\left[w^{\tau}\right]_{A_{s}} \beta_{n}^{s / r^{\prime}} \leq C_{1}\left[w^{\tau}\right]_{A_{s}}
$$

where $C_{1}$ is independent of $w$ and $b$. Now we return to (2.1). By (2.6) and (2.8), we get for $1<p<\infty$ and $w^{\tau} \in A_{s}$,

$$
\left\|V_{\rho} \mathcal{T}_{b}(f)\right\|_{L^{p}(w)} \leq \frac{C}{2 \pi \varepsilon} \int_{0}^{2 \pi}\left\|h_{\theta}\right\|_{L^{p}\left(e^{p b(\cdot) \varepsilon \cos \theta} w\right)} d \theta \leq C\|b\|_{*}\|f\|_{L^{p}(w)}
$$

Thus we complete the proof of Theorem 1.1.

\section{Proof of Theorem 1.6}

As having appeared in the proof of most of variational inequalities (in particular see [35]), we shall show the desired estimate by proving separately the long and short variational estimates. That is, we are reduced to show

$$
\left\|V_{\rho}\left(\left\{T_{\Omega, 2^{k} ; b} f\right\}_{k}\right)\right\|_{L^{p}} \leq C\|b\|_{*}\|f\|_{L^{p}}
$$

and

$$
\left\|S_{2}\left(\mathcal{T}_{\Omega ; b} f\right)\right\|_{L^{p}} \leq C\|b\|_{*}\|f\|_{L^{p}}
$$

where

$$
S_{2}\left(\mathcal{T}_{\Omega ; b} f\right)(x)=\left(\sum_{j \in \mathbb{Z}}\left[V_{2, j}\left(\mathcal{T}_{\Omega ; b} f\right)(x)\right]^{2}\right)^{1 / 2}
$$

with

$$
V_{2, j}\left(\mathcal{T}_{\Omega ; b} f\right)(x)=\left(\sup _{2^{j} \leq t_{0}<\cdots<t_{N}<2^{j+1}} \sum_{l=0}^{N}\left|T_{\Omega, t_{k+1} ; b} f(x)-T_{\Omega, t_{k} ; b} f(x)\right|^{2}\right)^{1 / 2} .
$$

To deal with the long variation (3.1), we use weighted estimates obtained in our previous paper [10] together with some Littlewood-Paley type estimates involving commutators, in addition to Theorem 1.1. While to handle with the short variation (3.2), we exploit Bony decomposition and paraproduct estimates, as well as Fefferman-Stein inequality for rough maximal functions. 


\subsection{Proof of Theorem (3.1)}

In this subsection, we give the proof of (3.1) and in the next subsection we deal with (3.2). Let us begin with one definition. For $j \in \mathbb{Z}$, let $\nu_{j}(x)=\frac{\Omega(y)}{|y|^{n}} \chi_{\left\{2^{j} \leq|x|<2^{j+1}\right\}}(x)$, then

$$
\nu_{j} * f(x)=\int_{2^{j} \leq|y|<2^{j+1}} \frac{\Omega(y)}{|y|^{n}} f(x-y) d y .
$$

Let $\phi \in \mathscr{S}\left(\mathbb{R}^{n}\right)$ be a radial function such that $\hat{\phi}(\xi)=1$ for $|\xi| \leq 2$ and $\hat{\phi}(\xi)=0$ for $|\xi|>4$. We have the following decomposition

$$
T_{\Omega, 2^{k}} f=\phi_{k} * T_{\Omega} f+\sum_{s \geq 0}\left(\delta_{0}-\phi_{k}\right) * \nu_{k+s} * f-\phi_{k} * \sum_{s<0} \nu_{k+s} * f
$$

where $\phi_{k}$ satisfies $\widehat{\phi_{k}}(\xi)=\hat{\phi}\left(2^{k} \xi\right), \delta_{0}$ is the Dirac measure at 0 and $s \in \mathbb{N} \cup\{0\}$. Then we have

$$
\begin{aligned}
T_{\Omega, 2^{k} ; b} f & =\left(\phi_{k} * T_{\Omega}\right)_{b} f+\left(\sum_{s \geq 0}\left(\delta_{0}-\phi_{k}\right) * \nu_{k+s}\right)_{b} f-\left(\phi_{k} * \sum_{s<0} \nu_{k+s}\right)_{b} f \\
& :=T_{k ; b}^{1} f+T_{k ; b}^{2} f-T_{k ; b}^{3} f .
\end{aligned}
$$

Let $\mathscr{T}_{b}^{i} f$ denote the family $\left\{T_{k ; b}^{i} f\right\}_{k \in \mathbb{Z}}$ for $i=1,2,3$. Obviously, to show (3.1) it suffices to prove the following inequalities:

$$
\left\|V_{\rho}\left(\mathscr{T}_{b}^{i} f\right)\right\|_{L^{p}} \leq C\|b\|_{*}\|f\|_{L^{p}}, \quad 1<p<\infty, \quad i=1,2,3 .
$$

Estimate of (3.3) for $i=1$. We need the following two lemmas.

Lemma 3.1. ([18]) Let $\phi_{k}$ be given above, set $\mathscr{U} f=\left\{\phi_{k} * f\right\}_{k}$. Then for $1<p<\infty$ and $2<\rho<\infty$,

$$
\left\|V_{\rho}(\mathscr{U} f)\right\|_{L^{p}} \leq C\|f\|_{L^{p}} .
$$

In [10, we proved that for $2<\rho<\infty, 1<p<\infty$ and $w \in A_{p}, V_{\rho} \mathscr{U}$ is bounded on $L^{p}(w)$. Then apply Theorem 1.1, we get

Lemma 3.2. For $k \in \mathbb{Z}$, let $\phi_{k}$ be given above and $b \in B M O\left(\mathbb{R}^{n}\right)$. Denote by $\Phi_{k} f=\phi_{k} * f, k \in \mathbb{Z}$. Let $\mathscr{U}_{b} f=\left\{\Phi_{k ; b} f\right\}_{k}$. Then for $1<p<\infty$ and $w \in A_{p}$,

$$
\left\|V_{\rho} \mathscr{U}_{b}(f)\right\|_{L^{p}(w)} \leq C\|f\|_{L^{p}(w)} .
$$

Write

$$
T_{k ; b}^{1} f=\Phi_{k ; b} T_{\Omega} f+\Phi_{k} T_{\Omega ; b} f .
$$

Then combining Lemma 3.1 with Lemma 3.2, the $L^{p}$-boundedness of $T_{\Omega}$ (see [7]) and $\left[b, T_{\Omega}\right]$ (see [29]), we can get the following estimate easily

$$
\begin{aligned}
\left\|V_{q}\left(\mathscr{T}_{b}^{1} f\right)\right\|_{L^{p}} & \leq\left\|V_{q}\left(\left\{\Phi_{k}\left(T_{\Omega ; b} f\right)\right\}_{k}\right)\right\|_{L^{p}}+\left\|V_{q}\left(\left\{\Phi_{k ; b}\left(T_{\Omega} f\right)\right\}_{k}\right)\right\|_{L^{p}} \\
& \leq C\left(\left\|T_{\Omega ; b} f\right\|_{L^{p}}+\|b\|_{*}\left\|T_{\Omega} f\right\|_{L^{p}}\right) \\
& \leq C\|\Omega\|_{L\left(\log ^{+} L\right)^{2}\left(\mathbf{S}^{n-1}\right)}\|b\|_{*}\|f\|_{L^{p}}
\end{aligned}
$$

Estimate of (3.3) for $i=2$. We first give two lemmas will be used in the following arguments. 
Lemma 3.3. ([29]) Let $m \in C_{0}^{\infty}\left(\mathbb{R}^{n}\right)$ and supp $m \subset\{|\xi| \leq 2 \sigma\}$ for some $\sigma \in(0, \infty)$. Suppose $m$ satisfies

$$
\|m\|_{L^{\infty}} \leq C 2^{-\gamma s} \min \left\{\sigma, \sigma^{-\lambda}\right\}, \quad\|\nabla m\|_{L^{\infty}} \leq C 2^{s}
$$

for some constants $C, \lambda, \gamma>0$ and $s \in \mathbb{N}$. Let $T_{m}$ be the multiplier operator defined by $\widehat{T_{m} f}(\xi)=$ $m(\xi) \widehat{f}(\xi)$. Moreover, for $b \in B M O$ and $u \in \mathbb{N}$, denote by $T_{m ; b, u} f(x)=T_{m}\left((b(x)-b(\cdot))^{u} f\right)(x)$ the $u$-th order commutator of $T_{m}$. Then for any $0<v<1$, there exist positive constants $C=C(n, v)$ and $\beta \in(0,1)$ such that

$$
\left\|T_{m ; b, u} f\right\|_{L^{2}} \leq C 2^{-\beta s} \min \left\{\sigma^{v}, \sigma^{-\lambda v}\right\}\|b\|_{*}^{u}\|f\|_{L^{2}} .
$$

Lemma 3.4. (see [29]). Let $b \in B M O\left(\mathbb{R}^{n}\right)$. Then, for $1<p<\infty$ and $f \in L^{p}\left(\mathbb{R}^{n}\right)$, we have

$$
\begin{aligned}
& \left\|\left(\sum_{l \in \mathbb{Z}}\left|\Delta_{l ; b, u} f\right|^{2}\right)^{1 / 2}\right\|_{L^{p}} \leq C(n, p)\|b\|_{*}^{u}\|f\|_{L^{p}} \\
& \left\|\left(\sum_{l \in \mathbb{Z}}\left|\Delta_{l ; b, u}^{2} f\right|^{2}\right)^{1 / 2}\right\|_{L^{p}} \leq C(n, p)\|b\|_{*}^{u}\|f\|_{L^{p}} .
\end{aligned}
$$

Let $E_{0}=\left\{x^{\prime} \in \mathbf{S}^{n-1}:\left|\Omega\left(x^{\prime}\right)\right|<2\right\}$ and $E_{d}=\left\{x^{\prime} \in \mathbf{S}^{n-1}: 2^{d} \leq\left|\Omega\left(x^{\prime}\right)\right|<2^{d+1}\right\}$ for positive integer $d$. For $d \geq 0$, let

$$
\Omega_{d}\left(y^{\prime}\right)=\Omega\left(y^{\prime}\right) \chi_{E_{d}}\left(y^{\prime}\right)-\frac{1}{\left|\mathbf{S}^{n-1}\right|} \int_{E_{d}} \Omega\left(x^{\prime}\right) d \sigma\left(x^{\prime}\right) .
$$

Since $\Omega$ satisfies (1.11), then

$$
\int_{\mathbf{S}^{n-1}} \Omega_{d}\left(y^{\prime}\right) d \sigma\left(y^{\prime}\right)=0 \text { for } d \geq 0
$$

and $\Omega\left(y^{\prime}\right)=\sum_{d \geq 0} \Omega_{d}\left(y^{\prime}\right)$. Set

$$
\nu_{j, d}(x)=\frac{\Omega_{d}(x)}{|x|^{n}} \chi_{\left\{2^{j} \leq|x|<2^{j+1}\right\}}(x) .
$$

Then, by the Minkowski inequality, we get

$$
\begin{aligned}
V_{q} \mathscr{T}_{b}^{2}(f)(x) & \leq \sum_{s \geq 0}\left(\sum_{k \in \mathbb{Z}}\left|\left(\left(\delta_{0}-\phi_{k}\right) * \nu_{k+s}\right)_{b} f(x)\right|^{2}\right)^{1 / 2} \\
& \leq \sum_{s \geq 0} \sum_{d \geq 0}\left(\sum_{k \in \mathbb{Z}}\left|\left(\left(\delta_{0}-\phi_{k}\right) * \nu_{k+s, d}\right)_{b} f(x)\right|^{2}\right)^{1 / 2}
\end{aligned}
$$

Let $\varphi \in C_{0}^{\infty}\left(\mathbb{R}^{n}\right)$ be a radial function such that $0 \leq \varphi \leq 1$, supp $\varphi \subset\{1 / 2 \leq|\xi| \leq 2\}$ and $\sum_{l \in \mathbb{Z}} \varphi^{2}\left(2^{-l} \xi\right)=1$ for $|\xi| \neq 0$. Define the multiplier $\Delta_{l}$ by $\widehat{\Delta_{l} f}(\xi)=\varphi\left(2^{-l} \xi\right) \hat{f}(\xi)$. By the Minkowski inequality again, we get

$$
V_{\rho} \mathscr{T}_{b}^{2}(f)(x) \leq \sum_{l \in \mathbb{Z}} \sum_{s \geq 0} \sum_{d \geq 0}\left(\sum_{k \in \mathbb{Z}}\left|\left(\left(\delta_{0}-\phi_{k}\right) * \nu_{k+s, d} * \Delta_{l-k}^{2}\right)_{b} f(x)\right|^{2}\right)^{1 / 2} .
$$


We set

$$
m_{s, k, d}(\xi)=\left(1-\widehat{\phi_{k}}(\xi)\right) \widehat{\nu_{k+s, d}}(\xi), \quad m_{s, k, d}^{l}(\xi)=\left(1-\widehat{\phi_{k}}(\xi)\right) \widehat{\nu_{k+s, d}}(\xi) \varphi\left(2^{k-l} \xi\right)
$$

Define multipliers $F_{s, k, d}$ and $F_{s, k, d}^{l}$, respectively by

$$
\widehat{F_{s, k, d} f}(\xi)=m_{s, k, d}(\xi) \widehat{f}(\xi)
$$

and

$$
\widehat{F_{s, k, d}^{l} f}(\xi)=m_{s, k, d}^{l}(\xi) \widehat{f}(\xi)
$$

Then

$$
V_{\rho} \mathscr{T}_{b}^{2}(f)(x) \leq \sum_{l \in \mathbb{Z}} \sum_{s \geq 0} \sum_{d \geq 0}\left(\sum_{k \in \mathbb{Z}}\left|\left(F_{s, k, d} \Delta_{l-k}^{2}\right)_{b} f(x)\right|^{2}\right)^{1 / 2} .
$$

If we can prove that

$$
\left\|\left(\sum_{k \in \mathbb{Z}}\left|\left(F_{s, k, d} \Delta_{l-k}^{2}\right)_{b} f\right|^{2}\right)^{1 / 2}\right\|_{L^{2}} \leq C\left\|\Omega_{d}\right\|_{L^{\infty}\left(\mathbf{S}^{n-1}\right)} 2^{-\beta s} 2^{-\theta|l|}\|b\|_{*}\|f\|_{L^{2}}
$$

and for $1<p<\infty$

$$
\left\|\left(\sum_{k \in \mathbb{Z}}\left|\left(F_{s, k, d} \Delta_{l-k}^{2}\right)_{b} f\right|^{2}\right)^{1 / 2}\right\|_{L^{p}} \leq C\left\|\Omega_{d}\right\|_{L \log ^{+} L\left(\mathbf{S}^{n-1}\right)}\|b\|_{*}\|f\|_{L^{p}},
$$

then we may finish the estimate of (3.3) for $i=2$. In fact, interpolating (3.5) and (3.6), we get for $0<\theta_{0}, \beta_{0}<1$,

$$
\left\|\left(\sum_{k \in \mathbb{Z}}\left|\left(F_{s, k, d} \Delta_{l-k}^{2}\right)_{b} f\right|^{2}\right)^{1 / 2}\right\|_{L^{p}} \leq C 2^{-\beta_{0} s} 2^{-\theta_{0}|l|}\|b\|_{*}\left\|\Omega_{d}\right\|_{L^{\infty}\left(\mathbf{S}^{n-1}\right)}\|f\|_{L^{p}}, \quad 1<p<\infty .
$$

Taking a large positive integer $N$, such that $N>\max \left\{2 \theta_{0}^{-1}, 2 \beta_{0}^{-1}\right\}$. We have

$$
\begin{aligned}
\left\|V_{\rho} \mathscr{T}_{b}^{2}(f)\right\|_{L^{p}} & \leq \sum_{d \geq 0} \sum_{l \in \mathbb{Z}}\left(\sum_{0 \leq s<N d}+\sum_{s>N d}\right)\left\|\left(\sum_{k \in \mathbb{Z}}\left|\left(F_{s, k, d} \Delta_{l-k}^{2}\right)_{b} f\right|^{2}\right)^{1 / 2}\right\|_{L^{p}} \\
& :=J_{1}+J_{2} .
\end{aligned}
$$

For $J_{1}$, by (3.6) and (3.7), we get

$$
\begin{aligned}
J_{1} & \leq C\|b\|_{*}\|f\|_{L^{p}} \sum_{d \geq 0} \sum_{0 \leq s<N d}\left(\sum_{0 \leq|l|<N d} d 2^{d} \sigma\left(E_{d}\right)+\sum_{|l|>N d} 2^{d} 2^{-\theta_{0}|l|}\right) \\
& \leq C\|b\|_{*}\|f\|_{L^{p}}\left(\sum_{d \geq 0} d^{3} 2^{d} \sigma\left(E_{d}\right)+\sum_{d \geq 0} d 2^{\left(1-\beta_{0} N\right) d}\right) \\
& \leq C\left(\|\Omega\|_{L\left(\log ^{+} L\right)^{3}\left(\mathbf{S}^{n-1}\right)}+1\right)\|b\|_{*}\|f\|_{L^{p}} .
\end{aligned}
$$


For $J_{2}$, using (3.7), we get

$$
\begin{aligned}
J_{3} & \leq C\|b\|_{*} \sum_{d \geq 0} 2^{d} \sum_{s>N d} 2^{-\beta_{0} s}\left(\sum_{|l|<N d}+\sum_{|l| \geq N d} 2^{-\theta_{0}|l|}\right)\|f\|_{L^{p}} \\
& \leq C\|b\|_{*} \sum_{d \geq 0}\left(d 2^{\left(1-\beta_{0} N\right) d}+2^{\left(1-\beta_{0} N-\theta_{0} N\right) d}\right)\|f\|_{L^{p}} \\
& \leq C\|b\|_{*}\|f\|_{L^{p}} .
\end{aligned}
$$

Finally, combining above two estimates, we get for $1<p<\infty$

$$
\left\|V_{\rho} \mathscr{T}_{b}^{2}(f)\right\|_{L^{p}} \leq C\left(1+\|\Omega\|_{L\left(\log ^{+} L\right)^{3}\left(\mathbf{S}^{n-1}\right)}\right)\|b\|_{*}\|f\|_{L^{p}}
$$

We therefore finish the estimate of $(3.3)$ for $i=2$.

Now we return to prove (3.5) and (3.6). We first prove (3.5). Note that $F_{s, k, d}^{l}=F_{s, k, d} \Delta_{l-k}$. We write

$$
\left(F_{s, k, d} \Delta_{l-k}^{2}\right)_{b} f=\sum_{u=0}^{1} F_{s, k, d ; b, u}^{l} \Delta_{l-k ; b, 1-u} f .
$$

Therefore

$$
\left\|\left(\sum_{k \in \mathbb{Z}}\left|\left(F_{s, k, d} \Delta_{l-k}^{2}\right)_{b} f\right|^{2}\right)^{1 / 2}\right\|_{L^{2}} \leq \sum_{u=0}^{1}\left\|\left(\sum_{k \in \mathbb{Z}}\left|F_{s, k, d ; b, u}^{l} \Delta_{l-k ; b, 1-u} f\right|^{2}\right)^{1 / 2}\right\|_{L^{2}} .
$$

To proceed with the estimate (3.8), we define multiplier $\widetilde{F}_{s, k, d}^{l}$ by $\widehat{\widetilde{F}_{s, k, d}^{l} f}(\xi)=m_{s, k, d}^{l}\left(2^{-k} \xi\right) \widehat{f}(\xi)$ and $\widetilde{F}_{s, k, d ; b, u}^{l}$ is the $u$-th commutator of $\widetilde{F}_{s, k, d}^{l}$. Recall that $m_{s, k, d}^{l}(\xi)=\left(1-\widehat{\phi_{k}}(\xi)\right) \widehat{\nu_{k+s, d}}(\xi) \varphi\left(2^{k-l} \xi\right)$. Since supp $\left(1-\widehat{\phi_{k}}\right) \widehat{\nu_{k+s, d}} \subset\left\{\xi:\left|2^{k} \xi\right|>1 / 2\right\}$, by a well-known Fourier transform estimate of Duoandikoetxea and Rubio de Francia (See (21], p.551-552), it is easy to show that there is a $\gamma \in(0,1)$ such that

$$
\left|\left(1-\widehat{\phi_{k}}(\xi)\right) \widehat{\nu_{k+s, d}}(\xi)\right| \leq C\left\|\Omega_{d}\right\|_{L^{\infty}\left(\mathbf{S}^{n-1}\right)} 2^{-s \gamma} \min \left\{\left|2^{k} \xi\right|,\left|2^{k} \xi\right|^{-\gamma}\right\}
$$

and

$$
\left|\nabla\left[\left(1-\widehat{\phi_{k}}(\xi)\right) \widehat{\nu_{k+s, d}}(\xi)\right]\right| \leq C\left\|\Omega_{d}\right\|_{L^{\infty}\left(\mathbf{S}^{n-1}\right)} 2^{k} 2^{s} .
$$

As a result, we have the following estimates

$$
\begin{gathered}
\operatorname{supp} m_{s, k, d}^{l}\left(2^{-k} \xi\right) \subset\left\{|\xi| \leq 2^{l}\right\} \\
\left|m_{s, k, d}^{l}\left(2^{-k} \xi\right)\right| \leq C\left\|\Omega_{d}\right\|_{L^{\infty}\left(\mathbf{S}^{n-1}\right)} 2^{-\gamma s} \min \left\{2^{l}, 2^{-\gamma l}\right\},
\end{gathered}
$$

and

$$
\left|\nabla m_{s, k, d}^{l}\left(2^{-k} \xi\right)\right| \leq C\left\|\Omega_{d}\right\|_{L^{\infty}\left(\mathbf{S}^{n-1}\right)} 2^{s}
$$


Applying Lemma 3.3 with $\sigma=2^{l}$ to (3.9)-(3.11), there exist constants $\beta \in(0,1)$ and $\theta \in(0,1)$ such that

$$
\left\|\widetilde{F}_{s, k, d ; b, u}^{l} f\right\|_{L^{2}} \leq C\left\|\Omega_{d}\right\|_{L^{\infty}\left(\mathbf{S}^{n-1}\right)}\|b\|_{*}^{u} 2^{-\beta s} 2^{-\theta|l|}\|f\|_{L^{2}}, \quad \text { for } \quad l \in \mathbb{Z} \quad \text { and } s \geq 0 .
$$

Further, the dilation-invariance implies

$$
\left\|F_{s, k, d ; b, u}^{l} f\right\|_{L^{2}} \leq C\left\|\Omega_{d}\right\|_{L^{\infty}\left(\mathbf{S}^{n-1}\right)}\|b\|_{*}^{u} 2^{-\beta s} 2^{-\theta|l|}\|f\|_{L^{2}}, \quad \text { for } l \in \mathbb{Z} \text { and } s \geq 0 .
$$

Then by (3.12) and Lemma 3.4, we get

$$
\begin{aligned}
\left\|\left(\sum_{k \in \mathbb{Z}}\left|\left(F_{s, k, d} \Delta_{l-k}^{2}\right)_{b} f\right|^{2}\right)^{1 / 2}\right\|_{L^{2}} & \leq C \sum_{u=0}^{1}\left\|\Omega_{d}\right\|_{L^{\infty}\left(\mathbf{S}^{n-1}\right)} 2^{-\beta s} 2^{-\theta|l|}\|b\|_{*}^{u}\left\|\left(\sum_{k \in \mathbb{Z}}\left|\Delta_{l-k ; b, 1-u} f\right|^{2}\right)^{1 / 2}\right\|_{L^{2}} \\
& \leq C\left\|\Omega_{d}\right\|_{L^{\infty}\left(\mathbf{S}^{n-1}\right)} 2^{-\beta s} 2^{-\theta|l|}\|b\|_{*}\|f\|_{L^{2}} .
\end{aligned}
$$

This gives (3.5). Secondly, we turn to prove (3.6). Write

$$
\left(F_{s, k, d} \Delta_{l-k}^{2}\right)_{b} f(x)=\sum_{u=0}^{1} F_{s, k, d ; b, u} \Delta_{l-k ; b, 1-u}^{2} f .
$$

By the Minkowski inequality, we get

$$
\left\|\left(\sum_{k \in \mathbb{Z}}\left|\left(F_{s, k, d} \Delta_{l-k}^{2}\right)_{b} f\right|^{2}\right)^{1 / 2}\right\|_{L^{p}} \leq \sum_{u=0}^{1}\left\|\left(\sum_{k \in \mathbb{Z}}\left|F_{s, k, d ; b, u} \Delta_{l-k ; b, 1-u}^{2} f\right|^{2}\right)^{1 / 2}\right\|_{L^{p}} .
$$

To proceed with the above estimate, we need the following lemma, which can be proved as that in $([21$, p. 544]).

Lemma 3.5. Suppose that $\left\{\sigma_{j}\right\}_{j \in \mathbb{Z}}$ is a sequence of finite Borel measures, $T_{j} f=\sigma_{j} * f$ and $\bar{T}_{j} f=\left|\sigma_{j}\right| * f$ for any $j \in \mathbb{Z}$. Let $b \in B M O$ and $T_{j ; b, u} f(x)=T_{j}\left((b(x)-b(\cdot))^{u} f\right)(x), u \in \mathbb{N}$. If the maximal operator $T_{b, u}^{*}(f)=\sup _{j \in \mathbb{Z}}\left|\bar{T}_{j ; b, u} f\right|$ is bounded on $L^{p_{0}}$ for any fixed $1<p_{0}<\infty$, then for any $1<p<\infty$,

$$
\left\|\left(\sum_{j \in \mathbb{Z}}\left|T_{j ; b, u} g_{j}\right|^{2}\right)^{1 / 2}\right\|_{L^{p}} \leq C\|b\|_{*}^{u}\left\|\left(\sum_{j \in \mathbb{Z}}\left|g_{j}\right|^{2}\right)^{1 / 2}\right\|_{L^{p}} .
$$

Recall that $F_{s, k, d} f(x)=\left(\delta_{0}-\phi_{k}\right) * \nu_{k+s} * f(x)$. Define by $\bar{F}_{s, k, d} f(x)=\left|\left(\delta_{0}-\phi_{k}\right) * \nu_{k+s}\right| * f(x)$. Since for some $1<p_{0}<\infty$,

$$
\left\|\sup _{k \in \mathbb{Z}}\left|\bar{F}_{s, k, d ; b, 1} f\right|\right\|_{L^{p_{0}}} \leq\left\|\Omega_{d}\right\|_{L \log ^{+} L\left(\mathbf{S}^{n-1}\right)}\|b\|_{*}\|f\|_{L^{p_{0}}}
$$

and

$$
\left\|\sup _{k \in \mathbb{Z}}\left|\bar{F}_{s, k, d} f\right|\right\|_{L^{p_{0}}} \leq\left\|\Omega_{d}\right\|_{L^{1}\left(\mathbf{S}^{n-1}\right)}\|f\|_{L^{p_{0}}}
$$


(see [29, 24]). So by (3.13), Lemma 3.5] and Lemma 3.4, we get for $1<p<\infty$,

$$
\left\|\left(\sum_{k \in \mathbb{Z}}\left|\left(F_{s, k, d} \Delta_{l-k}^{2}\right)_{b} f\right|^{2}\right)^{1 / 2}\right\|_{L^{p}} \leq C\left\|\Omega_{d}\right\|_{L \log ^{+} L\left(\mathbf{S}^{n-1}\right)}\|b\|_{*}\|f\|_{L^{p}},
$$

which gives (3.6).

Estimate of (3.3) for $i=3$. We have the following pointwise estimate

$$
V_{\rho} \mathscr{T}_{b}^{3}(f)(x) \leq \sum_{s<0}\left(\sum_{k \in \mathbb{Z}}\left|\left(\phi_{k} * \nu_{k+s}\right)_{b} f(x)\right|^{2}\right)^{1 / 2}
$$

The proofs are essentially similar to the proof of (3.3) for $i=2$. More precisely, we need to give the estimates on the left hand side of (3.5)-(3.6) with replacing $\left(\delta_{0}-\phi_{k}\right) * \nu_{k+s}$ by $\phi_{k} * \nu_{k+s}$. Since supp $\widehat{\phi_{k}} \widehat{\nu_{k+s}} \subset\left\{\xi:\left|2^{k} \xi\right|<1\right\}$ and $\Omega$ satisfies (1.11), then it is easy to see that

$$
\left|\widehat{\phi_{k} \nu_{k+s}}(\xi)\right| \leq C 2^{s}\|\Omega\|_{L^{1}\left(\mathbf{S}^{n-1}\right)} \min \left\{\left|2^{k} \xi\right|,\left|2^{k} \xi\right|^{-1}\right\}
$$

and

$$
\left|\nabla \widehat{\nu_{k+s}}(\xi)\right| \leq C 2^{(k+s)}\|\Omega\|_{L^{1}\left(\mathbf{S}^{n-1}\right)} .
$$

Set

$$
R_{s, k}(\xi)=\widehat{\phi_{k}}(\xi) \widehat{\nu_{k+s}}(\xi), \quad R_{s, k}^{l}(\xi)=R_{s, k}(\xi) \varphi\left(2^{k-l} \xi\right) .
$$

Using the two above inequalities, we have the following estimate

$$
\begin{gathered}
\operatorname{supp}_{s, k}^{l}\left(2^{-k} \xi\right) \subset\left\{|\xi| \leq 2^{l}\right\}, \\
\left|R_{s, k}^{l}\left(2^{-k} \xi\right)\right| \leq C 2^{s} \min \left\{2^{l}, 2^{-l}\right\}\|\Omega\|_{L^{1}\left(\mathbf{S}^{n-1}\right)}
\end{gathered}
$$

and

$$
\left|\nabla\left(R_{s, k}^{l}\left(2^{-k} \xi\right)\right)\right| \leq C 2^{s}\|\Omega\|_{L^{1}\left(\mathbf{S}^{n-1}\right)} .
$$

Then apply Lemma 3.3 and the same arguments of the proofs of (3.3) for $i=2$, then the right hand side of (3.5) is controlled by $C 2^{s} 2^{-\theta|l|}\|\Omega\|_{L^{1}\left(\mathbf{S}^{n-1}\right)}\|b\|_{*}\|f\|_{L^{2}}$ for $\theta>0$. It is also easy to get the same estimates in the right hand side of (3.6) by using Lemma 3.4, Lemma 3.5 and the results in [29, 24]. Then we get for $1<p<\infty$

$$
\left\|V_{\rho} \mathscr{T}_{b}^{3}(f)\right\|_{L^{p}} \leq C\|\Omega\|_{L \log ^{+} L\left(\mathbf{S}^{n-1}\right)}\|b\|_{*}\|f\|_{L^{p}}
$$

We therefore finish the proof of (3.3) for $i=3$. 


\subsection{Proof of 3.2}

In the section, we give the proof of (3.2). For $t \in[1,2)$, we define $\nu_{0, t}$ as

$$
\nu_{0, t}(x)=\frac{\Omega\left(x^{\prime}\right)}{|x|^{n}} \chi_{\{t \leq|x| \leq 2\}}(x)
$$

and $\nu_{j, t}(x)=2^{-j n} \nu_{0, t}\left(2^{-j} x\right)$ for $j \in \mathbb{Z}$. Denote $T_{j, t}$ by $T_{j, t} f(x)=\nu_{j, t} * f(x)$ and $T_{j, t ; b}$ by $\left.T_{j, t ; b} f(x)=T_{j, t}(b(x)-b(\cdot)) f\right)(x)$. Observe that $V_{2, j}\left(\mathcal{T}_{b} f\right)(x)$ is just the strong 2-variation function of the family $\left\{T_{j, t ; b} f(x)\right\}_{t \in[1,2)}$ and $\sum_{k \in \mathbb{Z}} \Delta_{k}^{2}=\mathcal{I}$ (identity operator), hence

$$
\begin{aligned}
S_{2}\left(\mathcal{T}_{b} f\right)(x) & =\left(\sum_{j \in \mathbb{Z}}\left|V_{2, j}\left(\mathcal{T}_{b} f\right)(x)\right|^{2}\right)^{\frac{1}{2}}=\left(\sum_{j \in \mathbb{Z}}\left\|\left\{T_{j, t ; b} f(x)\right\}_{t \in[1,2)}\right\|_{V_{2}}^{2}\right)^{\frac{1}{2}} \\
& \leq \sum_{k \in \mathbb{Z}}\left(\sum_{j \in \mathbb{Z}}\left\|\left\{\left(T_{j, t} \Delta_{k-j}^{2}\right)_{b} f(x)\right\}_{t \in[1,2)}\right\|_{V_{2}}^{2}\right)^{\frac{1}{2}} \\
& :=\sum_{k \in \mathbb{Z}} S_{2, k}\left(\mathcal{T}_{b} f\right)(x) .
\end{aligned}
$$

For $d \geq 0$, set

$$
\nu_{j, t, d}(x)=\frac{\Omega_{d}(x)}{|x|^{n}} \chi_{\left\{2^{j} t \leq|x|<2^{j+1}\right\}}(x) .
$$

$T_{j, t, d}$ is defined as $T_{j, t}$ by replacing $\nu_{j, t}$ by $\nu_{j, t, d}$. Decompose $\Omega$ as in the estimate of (3.3) for $i=2$. Then,

$$
S_{2}\left(\mathcal{T}_{b} f\right)(x) \leq \sum_{d \geq 0} \sum_{k \in \mathbb{Z}}\left(\sum_{j \in \mathbb{Z}}\left\|\left\{\left(T_{j, t, d} \Delta_{k-j}^{2}\right)_{b} f(x)\right\}_{t \in[1,2)}\right\|_{V_{2}}^{2}\right)^{\frac{1}{2}}:=\sum_{d \geq 0} \sum_{k \in \mathbb{Z}} S_{2, k, d}\left(\mathcal{T}_{b} f\right)(x) .
$$

Proposition 3.6. For $d \geq 0$ and $k \in \mathbb{Z}$, following conclusions hold:

(i) There exist a constant $\theta \in(0,1)$ such that

$$
\left\|S_{2, k, d}\left(\mathcal{T}_{b} f\right)\right\|_{L^{2}} \leq C\left\|\Omega_{d}\right\|_{L^{\infty}\left(\mathbf{S}^{n-1}\right)} 2^{-\frac{\theta}{2}|k|}\|b\|_{*}\|f\|_{L^{2}}
$$

(ii) For $1<p<\infty$,

$$
\left\|S_{2, k, d}\left(\mathcal{T}_{b} f\right)\right\|_{L^{p}} \leq C\left\|\Omega_{d}\right\|_{L \log ^{+} L\left(\mathbf{S}^{n-1}\right)}\|b\|_{*}\|f\|_{L^{p}} .
$$

The constants $C^{\prime} s$ in (3.14) and (3.15) are independent of $k$.

Using the same argument of (3.3) for $i=2$, we may finish the proof of (3.2) by using Proposition 3.6. We omit the details.

Proof of Proposition 3.6. Without loss of generality, we will use $S_{2, k}$ to replace with $S_{2, k, d}$. To deal with (3.14), we borrow the fact $\|\mathfrak{a}\|_{V_{2}} \leq\|\mathfrak{a}\|_{L^{2}}^{1 / 2}\left\|\mathfrak{a}^{\prime}\right\|_{L^{2}}^{1 / 2}$, where $\mathfrak{a}^{\prime}=\left\{\frac{d}{d t} a_{t}: t \in \mathbb{R}\right\}$. It is a special case of (39) in [35. Then,

$$
\begin{aligned}
{\left[S_{2, k}\left(\mathcal{T}_{b} f\right)(x)\right]^{2} } & \leq \sum_{j \in \mathbb{Z}}\left(\int_{1}^{2}\left|\left(T_{j, t} \Delta_{k-j}^{2}\right)_{b} f(x)\right|^{2} \frac{d t}{t}\right)^{1 / 2}\left(\int_{1}^{2}\left|\left(\frac{d}{d t} T_{j, t} \Delta_{k-j}^{2}\right)_{b} f(x)\right|^{2} \frac{d t}{t}\right)^{1 / 2} \\
& :=\sum_{j \in \mathbb{Z}} I_{1, k} f(x) \cdot I_{2, k} f(x) .
\end{aligned}
$$


By the Cauchy-Schwarz inequality, we have

$$
\left\|S_{2, k}\left(\mathcal{T}_{b} f\right)\right\|_{L^{2}}^{2} \leq\left\|I_{1, k} f\right\|_{L^{2}}\left\|I_{2, k} f\right\|_{L^{2}}
$$

We estimate $\left\|I_{1, k} f\right\|_{L^{2}}$ and $\left\|I_{2, k} f\right\|_{L^{2}}$, respectively. To estimate $\left\|I_{1, k} f\right\|_{L^{2}}$, we need the following estimates: for some $\gamma>0$,

$$
\left|\widehat{\nu_{j, t}}(\xi)\right| \leq C\|\Omega\|_{L^{\infty}\left(\mathbf{S}^{n-1}\right)} \min \left\{\left|2^{j} \xi\right|^{-\gamma}, 2^{j}|\xi|\right\}
$$

and

$$
\left|\nabla \widehat{\nu_{j, t}}(\xi)\right| \leq C\|\Omega\|_{L^{\infty}\left(\mathbf{S}^{n-1}\right)}
$$

uniformly in $t \in[1,2)$, which have been essentially proved in [21] and [29]. Similarly to the proof of (3.5), we get for some $v>0$,

$$
\left\|I_{1, k} f\right\|_{L^{2}} \leq C\|\Omega\|_{L^{\infty}\left(\mathbf{S}^{n-1}\right)}\|b\|_{*} \min \left\{2^{v k}, 2^{-\gamma v k}\right\}\|f\|_{L^{2}} .
$$

Next, we estimate $\left\|I_{2, k} f\right\|_{L^{2}}$. Write

$$
\left(\frac{d}{d t} T_{j, t} \Delta_{k-j}^{2}\right)_{b} f=\Delta_{k-j ; b} \frac{d}{d t} T_{j, t} \Delta_{k-j} f+\Delta_{k-j}\left(\frac{d}{d t} T_{j, t}\right)_{b} \Delta_{k-j} f+\Delta_{k-j} \frac{d}{d t} T_{j, t} \Delta_{k-j ; b} f .
$$

Thus we get

$$
\begin{aligned}
\left\|I_{2, k} f\right\|_{L^{2}} & \leq\left(\int_{1}^{2}\left\|\left(\sum_{j \in \mathbb{Z}}\left|\Delta_{k-j ; b} \frac{d}{d t} T_{j, t} \Delta_{k-j} f\right|^{2}\right)^{1 / 2}\right\|_{L^{2}}^{2} \frac{d t}{t}\right)^{1 / 2} \\
& +\left(\int_{1}^{2}\left\|\left(\sum_{j \in \mathbb{Z}}\left|\Delta_{k-j}\left(\frac{d}{d t} T_{j, t}\right)_{b} \Delta_{k-j} f\right|^{2}\right)^{1 / 2}\right\|_{L^{2}}^{2} \frac{d t}{t}\right)^{1 / 2} \\
& +\left(\int_{1}^{2}\left\|\left(\sum_{j \in \mathbb{Z}}\left|\Delta_{k-j} \frac{d}{d t} T_{j, t} \Delta_{k-j ; b} f\right|^{2}\right)^{1 / 2}\right\|_{L^{2}}^{2} \frac{d t}{t}\right)^{1 / 2} \\
& :=I+I I+I I I .
\end{aligned}
$$

To estimate $I, I I, I I I$, respectively, we need the following elementary fact

$$
\begin{aligned}
\frac{d}{d t} T_{j, t} h(x) & =\frac{d}{d t}\left[\int_{2^{j} t<|y| \leq 2^{j+1}} \frac{\Omega\left(y^{\prime}\right)}{|y|^{n}} h(x-y) d y\right] \\
& =\frac{d}{d t}\left[\int_{\mathbf{S}^{n-1}} \Omega\left(y^{\prime}\right) \int_{2^{j} t}^{2^{j+1}} \frac{1}{r} h\left(x-r y^{\prime}\right) d r d \sigma\left(y^{\prime}\right)\right] \\
& =-\frac{1}{t} \int_{\mathbf{S}^{n-1}} \Omega\left(y^{\prime}\right) h\left(x-2^{j} t y^{\prime}\right) d \sigma\left(y^{\prime}\right)
\end{aligned}
$$

and

$$
\left\|T_{j, t}^{*} h\right\|_{L^{2}} \leq C\|\Omega\|_{L^{1}\left(\mathbf{S}^{n-1}\right)}\|h\|_{L^{2}}
$$


where

$$
T_{j, t}^{*} h(x)=\int_{\mathbf{S}^{n-1}}\left|\Omega\left(y^{\prime}\right)\right|\left|h\left(x-2^{j} t y^{\prime}\right)\right| d \sigma\left(y^{\prime}\right)
$$

for $t \in[1,2)$. We now estimate $I$. Indeed, by (3.18), Lemma 3.4 and Littlewood-Paley theory, we have

$$
\begin{aligned}
I & \leq C\|b\|_{*}\left(\int_{1}^{2} \sum_{j \in \mathbb{Z}}\left\|T_{j, t}^{*} \Delta_{k-j} f\right\|_{L^{2}}^{2} \frac{d t}{t}\right)^{1 / 2} \\
& \leq C\|b\|_{*}\|\Omega\|_{L^{1}\left(\mathbf{S}^{n-1}\right)}\left\|\left(\sum_{j \in \mathbb{Z}}\left|\Delta_{k-j} f\right|^{2}\right)^{1 / 2}\right\|_{L^{2}} \\
& \leq C\|b\|_{*}\|\Omega\|_{L^{1}\left(\mathbf{S}^{n-1}\right)}\|f\|_{L^{2}} .
\end{aligned}
$$

Similarly, by (3.18), Lemma 3.4 and Littlewood-Paley theory, we get

$$
I I I \leq C\|b\|_{*}\|\Omega\|_{L^{1}\left(\mathbf{S}^{n-1}\right)}\|f\|_{L^{2}} .
$$

For $I I$, we will apply the Bony paraproduct to do this. Let $\varpi \in \mathscr{S}\left(\mathbb{R}^{n}\right)$ be a radial function satisfying $0 \leq \varpi \leq 1$ with its support is in the unit ball and $\varpi(\xi)=1$ for $|\xi| \leq \frac{1}{2}$. The function $\psi(\xi)=\varpi\left(\frac{\xi}{2}\right)-\varpi(\xi) \in \mathscr{S}\left(\mathbb{R}^{n}\right)$ supported on $\left\{\frac{1}{2} \leq|\xi| \leq 2\right\}$ and satisfies the identity $\sum_{j \in \mathbb{Z}} \psi\left(2^{-j} \xi\right)=1$ for $\xi \neq 0$. For $j \in \mathbb{Z}$, denote by $\Theta_{j}$ and $G_{j}$ the convolution operators whose the symbols are $\psi\left(2^{-j} \xi\right)$ and $\varpi\left(2^{-j} \xi\right)$, respectively. That is, $\Theta_{j}$ and $G_{j}$ are defined by $\widehat{\Theta_{j} f}(\xi)=\psi\left(2^{-j} \xi\right) \hat{f}(\xi)$ and $\widehat{G_{j} f}(\xi)=\varpi\left(2^{-j} \xi\right) \hat{f}(\xi)$ (see [24). The paraproduct of Bony between two functions $f, g$ is defined by

$$
\pi_{f}(g)=\sum_{j \in \mathbb{Z}}\left(\Theta_{j} f\right)\left(G_{j-3} g\right)
$$

At least formally, we have the following Bony decomposition

$$
f g=\pi_{f}(g)+\pi_{g}(f)+R(f, g) \quad \text { with } \quad R(f, g)=\sum_{i \in \mathbb{Z}} \sum_{|k-i| \leq 2}\left(\Theta_{i} f\right)\left(\Theta_{k} g\right) .
$$

Denote $f_{k, j}:=\Delta_{k-j} f$ and $T_{j, t}^{\prime} f:=\frac{d}{d t} T_{j, t} f$. By (3.19), we have

$$
\begin{aligned}
{\left[b, T_{j, t}^{\prime}\right] f_{k, j}(x) } & =b(x)\left(T_{j, t}^{\prime} f_{k, j}\right)(x)-T_{j, t}^{\prime}\left(b f_{k, j}\right)(x) \\
& =\left[\pi_{T_{j, t}^{\prime} f_{k, j}}(b)(x)-T_{j, t}^{\prime}\left(\pi_{f_{k, j}}(b)\right)(x)\right] \\
& +\left[R\left(b, T_{j, t}^{\prime} f_{k, j}\right)(x)-T_{j, t}^{\prime}\left(R\left(b, f_{k, j}\right)\right)(x)\right] \\
& +\left[\pi_{b}\left(T_{j, t}^{\prime} f_{k, j}\right)(x)-T_{j, t}^{\prime}\left(\pi_{b}\left(f_{k, j}\right)\right)(x)\right] .
\end{aligned}
$$


Thus

$$
\begin{aligned}
I I \leq & \left(\int_{1}^{2}\left\|\left(\sum_{j \in \mathbb{Z}}\left|\Delta_{k-j}\left[\pi_{T_{j, t}^{\prime} f_{k, j}}(b)-T_{j, t}^{\prime}\left(\pi_{f_{k, j}}(b)\right)\right]\right|^{2}\right)^{1 / 2}\right\|_{L^{2}}^{2} \frac{d t}{t}\right)^{1 / 2} \\
+ & \left(\int_{1}^{2}\left\|\left(\sum_{j \in \mathbb{Z}}\left|\Delta_{k-j}\left[R\left(b, T_{j, t}^{\prime} f_{k, j}\right)-T_{j, t}^{\prime}\left(R\left(b, f_{k, j}\right)\right)\right]\right|^{2}\right)^{1 / 2}\right\|_{L^{2}}^{2} \frac{d t}{t}\right)^{1 / 2} \\
& +\left(\int_{1}^{2}\left\|\left(\sum_{j \in \mathbb{Z}}\left|\Delta_{k-j}\left[\pi_{b}\left(T_{j, t}^{\prime} f_{k, j}\right)-T_{j, t}^{\prime}\left(\pi_{b}\left(f_{k, j}\right)\right)\right]\right|^{2}\right)^{1 / 2}\right\|_{L^{2}}^{2} \frac{d t}{t}\right)^{1 / 2} \\
:= & I I_{1}+I I_{2}+I I_{3} .
\end{aligned}
$$

We will estimate $I I_{i}, i=1,2,3$, respectively. For $I I_{1}$, note that $\Theta_{i} \Delta_{k-j} g=0$ for $g \in \mathscr{S}^{\prime}\left(\mathbb{R}^{n}\right)$ when $|i-(k-j)| \geq 3$, by (3.17), we get

$$
\begin{aligned}
& {\left[\pi_{T_{j, t}^{\prime} f_{k, j}}(b)(x)-T_{j, t}^{\prime}\left(\pi_{f_{k, j}}(b)\right)(x)\right] } \\
= & \sum_{i \in \mathbb{Z}}\left\{\left(T_{j, t}^{\prime} \Theta_{i} \Delta_{k-j} f\right)(x)\left(G_{i-3} b\right)(x)-T_{j, t}^{\prime}\left[\left(\Theta_{i} \Delta_{k-j} f\right)\left(G_{i-3} b\right)\right](x)\right\} \\
= & \sum_{|i-(k-j)| \leq 2} \frac{1}{t} \int_{\mathbf{S}^{n-1}} \Omega\left(y^{\prime}\right)\left(G_{i-3} b(x)-G_{i-3} b\left(x-2^{j} t y^{\prime}\right)\right)\left(\Theta_{i} \Delta_{k-j} f\right)\left(x-2^{j} t y^{\prime}\right) d \sigma\left(y^{\prime}\right) .
\end{aligned}
$$

To estimate the above inequality, we need the follow lemma.

Lemma 3.7. ([12]) For any fixed $0<\tau<1 / 2$, we have

$$
\left|G_{k} b(x)-G_{k} b(y)\right| \leq C \frac{2^{k \tau}}{\tau}|x-y|^{\tau}\|b\|_{*}
$$

where $C$ is independent of $k$ and $\tau$.

By Lemma 3.7, note that for $t \in[1,2)$, we get

$$
\begin{aligned}
& \left|\frac{1}{t} \int_{\mathbf{S}^{n-1}} \Omega\left(y^{\prime}\right)\left(G_{i-3} b(x)-G_{i-3} b\left(x-2^{j} t y^{\prime}\right)\right)\left(\Theta_{i} \Delta_{k-j} f\right)\left(x-2^{j} t y^{\prime}\right) d \sigma\left(y^{\prime}\right)\right| \\
& \leq C \frac{2^{(i+j) \tau}}{\tau}\|b\|_{*} \int_{\mathbf{S}^{n-1}}\left|\Omega\left(y^{\prime}\right) \|\left(\Theta_{i} \Delta_{k-j} f\right)\left(x-2^{j} t y^{\prime}\right)\right| d \sigma\left(y^{\prime}\right) \\
& \leq C \frac{2^{(i+j) \tau}}{\tau}\|b\|_{*} T_{j, t}^{*}\left(\Theta_{i} \Delta_{k-j} f\right)(x) .
\end{aligned}
$$

Then by (3.20)-(3.21), (3.18) and Littlewood-Paley theory, we have

$$
\begin{aligned}
I I_{1} & \leq C\|b\|_{*} \frac{2^{k \tau}}{\tau} \sum_{|l| \leq 2}\left(\int_{1}^{2}\left\|\left(\sum_{j \in \mathbb{Z}}\left|T_{j, t}^{*}\left(\Theta_{k-j+l} \Delta_{k-j} f\right)\right|^{2}\right)^{1 / 2}\right\|_{L^{2}}^{2} \frac{d t}{t}\right)^{1 / 2} \\
& \leq C \frac{2^{k \tau}}{\tau}\|b\|_{*}\|\Omega\|_{L^{1}\left(\mathbf{S}^{n-1}\right)}\|f\|_{L^{2}},
\end{aligned}
$$


where $C$ is independent of $k$ and $\tau$.

Next, we estimate $I I_{2}$. Clearly, $\Theta_{i+l} \Delta_{k-j} g=0$ for $g \in \mathscr{S}^{\prime}\left(\mathbb{R}^{n}\right)$ when $|l| \leq 2$ and $|i-(k-j)| \geq$ 8. Thus by (3.17),

$$
\begin{aligned}
& {\left[R\left(b, T_{j, t}^{\prime} f_{k, j}\right)(x)-T_{j, t}^{\prime}\left(R\left(b, f_{k, j}\right)\right)(x)\right] } \\
= & \sum_{i \in \mathbb{Z}} \sum_{|l| \leq 2}\left(\Theta_{i} b\right)(x)\left(T_{j, t}^{\prime} \Theta_{i+l} \Delta_{k-j} f\right)(x)-T_{j, t}^{\prime}\left(\sum_{i \in \mathbb{Z}} \sum_{|l| \leq 2}\left(\Theta_{i} b\right)\left(\Theta_{i+l} \Delta_{k-j} f\right)\right)(x) \\
= & \sum_{l=-2}^{2} \sum_{|i-(k-j)| \leq 7}\left(\left(\Theta_{i} b\right)(x)\left(T_{j, t}^{\prime} \Theta_{i+l} \Delta_{k-j} f\right)(x)-T_{j, t}^{\prime}\left(\left(\Theta_{i} b\right)\left(\Theta_{i+l} \Delta_{k-j} f\right)\right)(x)\right) \\
= & \sum_{l=-2}^{2} \sum_{|i-(k-j)| \leq 7} \frac{1}{t} \int_{\mathbf{S}^{n-1}} \Omega\left(y^{\prime}\right)\left(\Theta_{i} b(x)-\Theta_{i} b\left(x-2^{j} t y^{\prime}\right)\right) \Theta_{i+l} \Delta_{k-j} f\left(x-2^{j} t y^{\prime}\right) d \sigma\left(y^{\prime}\right) .
\end{aligned}
$$

Note that for $t \in[1,2)$, by $\sup _{i \in \mathbb{Z}}\left\|\Theta_{i}(b)\right\|_{L^{\infty}} \leq C\|b\|_{*}$ (see [24]), we get for any $h \in L^{p}\left(\mathbb{R}^{n}\right)$,

$$
\begin{aligned}
& \left|\frac{1}{t} \int_{\mathbf{S}^{n-1}} \Omega\left(y^{\prime}\right)\left(\Theta_{i} b(x)-\Theta_{i} b\left(x-2^{j} t y^{\prime}\right)\right) h\left(x-2^{j} t y^{\prime}\right) d \sigma\left(y^{\prime}\right)\right| \\
& \leq C \sup _{i \in \mathbb{Z}}\left\|\Theta_{i}(b)\right\|_{L^{\infty}} \int_{\mathbf{S}^{n-1}}\left|\Omega\left(y^{\prime}\right) \| h\left(x-2^{j} t y^{\prime}\right)\right| d \sigma\left(y^{\prime}\right) \\
& \leq C\|b\|_{*} T_{j, t}^{*} h(x) .
\end{aligned}
$$

Using (3.22)-(3.23), (3.18) and Littlewood-Paley theory, we have

$$
\begin{aligned}
I I_{2} & \leq C\|b\|_{*}\|\Omega\|_{L^{1}\left(\mathbf{S}^{n-1}\right)} \sum_{|l| \leq 7}\left(\int_{1}^{2}\left\|\left(\sum_{j \in \mathbb{Z}}\left|T_{j, t}^{*}\left(\Theta_{k-j+l} \Delta_{k-j} f\right)\right|^{2}\right)^{1 / 2}\right\|_{L^{2}}^{2} \frac{d t}{t}\right)^{1 / 2} \\
& \leq C\|b\|_{*}\|\Omega\|_{L^{1}\left(\mathbf{S}^{n-1}\right)}\|f\|_{L^{2}}
\end{aligned}
$$

Finally, we estimate $I I_{3}$. Note that $\Delta_{k-j}\left(\left(\Theta_{i} g\right)\left(G_{i-3} h\right)\right)=0$ for $g, h \in \mathscr{S}^{\prime}\left(\mathbb{R}^{n}\right)$ if $|i-(k-j)| \geq$ 5. Thus we get

$$
\begin{aligned}
& \Delta_{k-j}\left[\pi_{b}\left(T_{j, t}^{\prime} f_{k, j}\right)(x)-T_{j, t}^{\prime}\left(\pi_{b}\left(f_{k, j}\right)\right)\right](x) \\
= & \Delta_{k-j}\left(\sum_{i \in \mathbb{Z}}\left(\Theta_{i} b\right)\left(G_{i-3} T_{j, t}^{\prime} \Delta_{k-j} f\right)-T_{j, t}^{\prime}\left(\sum_{i \in \mathbb{Z}}\left(\Theta_{i} b\right)\left(G_{i-3} \Delta_{k-j} f\right)\right)\right)(x) \\
= & \sum_{|i-(k-j)| \leq 4} \Delta_{k-j}\left\{\left(\Theta_{i} b T_{j, t}^{\prime}\left(G_{i-3} \Delta_{k-j} f\right)\right)-T_{j, t}^{\prime}\left(\left(\Theta_{i} b\right)\left(G_{i-3} \Delta_{k-j} f\right)\right)\right\}(x) .
\end{aligned}
$$

Since

$$
\begin{aligned}
& \left(\Theta_{i} b T_{j, t}^{\prime}\left(G_{i-3} \Delta_{k-j} f\right)\right)-T_{j, t}^{\prime}\left(\left(\Theta_{i} b\right)\left(G_{i-3} \Delta_{k-j} f\right)\right)(x) \\
& =\frac{1}{t} \int_{\mathbf{S}^{n-1}} \Omega\left(y^{\prime}\right)\left(\Theta_{i} b(x)-\Theta_{i} b\left(x-2^{j} t y^{\prime}\right)\right) G_{i-3} \Delta_{k-j} f\left(x-2^{j} t y^{\prime}\right) d \sigma\left(y^{\prime}\right) .
\end{aligned}
$$


Thus, by (3.23), (3.18) and Littlewood-Paley theory, we get

$$
\begin{aligned}
I I_{3} & \leq C\|b\|_{*}\|\Omega\|_{L^{1}\left(\mathbf{S}^{n-1}\right)} \sum_{|l| \leq 4}\left(\int_{1}^{2}\left\|\left(\sum_{j \in \mathbb{Z}}\left|T_{j, t}^{*} G_{k-j+l-3} \Delta_{k-j} f\right|^{2}\right)^{1 / 2}\right\|_{L^{2}}^{2} \frac{d t}{t}\right)^{1 / 2} \\
& \leq C\|b\|_{*}\|\Omega\|_{L^{1}\left(\mathbf{S}^{n-1}\right)}\|f\|_{L^{2}} .
\end{aligned}
$$

Together with the estimates of $I I_{1}, I I_{2}$ and $I I_{3}$, we get

$$
I I \leq C \max \left\{2, \frac{2^{\tau k}}{\tau}\right\}\|b\|_{*}\|\Omega\|_{L^{1}\left(\mathbf{S}^{n-1}\right)}\|f\|_{L^{2}} \quad \text { for } \quad k \in \mathbb{Z},
$$

where $C$ is independent of $k$ and $\tau$. Taking $\tau=\frac{1}{|k|}$ in (3.24), we get

$$
I I \leq C(|k|+1)\|b\|_{*}\|\Omega\|_{L^{1}\left(\mathbf{S}^{n-1}\right)}\|f\|_{L^{2}} \quad \text { for } \quad k \in \mathbb{Z} .
$$

Combining this with the estimate of $I$ and $I I I$, we get

$$
\left\|I_{2, k} f\right\|_{L^{2}} \leq C(|k|+1)\|b\|_{*}\|\Omega\|_{L^{1}\left(\mathbf{S}^{n-1}\right)}\|f\|_{L^{2}} .
$$

Combining the estimates of (3.16) and (3.25), we get for some constant $\theta \in(0,1)$ and $k \in \mathbb{Z}$,

$$
\begin{aligned}
\left\|S_{2, k}\left(\mathcal{T}_{b} f\right)\right\|_{L^{2}}^{2} & \leq C \min \left\{2^{v k}, 2^{-\gamma v k}\right\}(1+|k|)\|b\|_{*}^{2}\|\Omega\|_{L^{\infty}\left(\mathbf{S}^{n-1}\right)}^{2}\|f\|_{L^{2}}^{2} \\
& \leq C 2^{-\theta|k|}\|b\|_{*}^{2}\|\Omega\|_{L^{\infty}\left(\mathbf{S}^{n-1}\right)}^{2}\|f\|_{L^{2}}^{2} .
\end{aligned}
$$

This finishes the proof of (3.14).

Proof of (3.15). Let

$$
B=\left\{\left\{a_{j, t}\right\}_{j \in \mathbb{Z}, t \in[1,2)}:\left\|a_{j, t}\right\|_{B}:=\left(\sum_{j \in \mathbb{Z}}\left\|a_{j, t}\right\|_{V_{2}}^{2}\right)^{1 / 2}<\infty\right\} .
$$

Clearly, $\left(B,\|\cdot\|_{B}\right)$ is a Banach space.Then,

$$
\begin{aligned}
S_{2, k}\left(\mathcal{T}_{b} f\right)(x) & =\left(\sum_{j \in \mathbb{Z}} \sup _{\substack{\left.t_{1}<\cdots<t_{N} \\
t_{l}, t_{l+1}\right] \subset[1,2)}} \sum_{l=1}^{N-1}\left|\left(T_{j, t_{l}} \Delta_{k-j}^{2}\right)_{b} f(x)-\left(T_{j, t_{l+1}} \Delta_{k-j}^{2}\right)_{b} f(x)\right|^{2}\right)^{\frac{1}{2}} \\
& =\left(\sum_{j \in \mathbb{Z}} \sup _{\substack{\left.t_{1}<\cdots<t_{N} \\
t_{l}, t_{l+1}\right] \subset[1,2)}} \sum_{l=1}^{N-1}\left|\left(T_{j, t_{l}, t_{l+1}} \Delta_{k-j}^{2}\right)_{b} f(x)\right|^{2}\right)^{\frac{1}{2}}
\end{aligned}
$$

where

$$
T_{j, t_{l}, t_{l+1}} f(x)=\int_{2^{j} t_{l}<|y| \leq 2^{j} t_{l+1}} f(x-y) \frac{\Omega(y)}{|y|^{n}} d y \text { and }\left[t_{l}, t_{l+1}\right] \subset[1,2) .
$$

Then we get

$$
S_{2, k}\left(\mathcal{T}_{b} f\right)(x) \leq \sum_{u=0}^{1}\left(\sum_{j \in \mathbb{Z}} \sup _{\substack{t_{1}<\cdots<t_{N} \\\left[t_{l}, t_{l+1}\right] \subset[1,2)}} \sum_{l=1}^{N-1}\left|T_{j, t_{l}, t_{l+1} ; b, u} \Delta_{k-j ; b, 1-u}^{2} f(x)\right|^{2}\right)^{\frac{1}{2}} .
$$


For $\left[t_{l}, t_{l+1}\right] \subset[1,2)$ and $u \in \mathbb{N}$, let

$$
\widetilde{T}_{j, t_{l}, t_{l+1} ; b, u} f(x)=\int_{2^{j} t_{l}<|x-y| \leq 2^{j} t_{l+1}}|f(y)||b(x)-b(y)|^{u} \frac{|\Omega(x-y)|}{|x-y|^{n}} d y .
$$

Then,

$$
\begin{aligned}
S_{2, k}\left(\mathcal{T}_{b} f\right)(x) & \leq \sum_{u=0}^{1}\left(\sum_{j \in \mathbb{Z}} \sup _{\substack{\left.t_{1}<\cdots<t_{N} \\
t_{l}, t_{l+1}\right] \subset[1,2)}} \sum_{l=1}^{N-1}\left|\widetilde{T}_{j, t_{l}, t_{l+1} ; b, u} \Delta_{k-j ; b, 1-u}^{2} f(x)\right|^{2}\right)^{\frac{1}{2}} \\
& =\sum_{u=0}^{1}\left(\sum_{j \in \mathbb{Z}} \sup _{\substack{\left.t_{1}<t_{N} \\
t_{1}, t_{N}\right] \subset[1,2)}}\left|\widetilde{T}_{j, t_{1}, t_{N} ; b, u}\left(\Delta_{k-j ; b, 1-u}^{2} f\right)(x)\right|^{2}\right)^{\frac{1}{2}} .
\end{aligned}
$$

Therefore, we get

$$
S_{2, k}\left(\mathcal{T}_{b} f\right)(x) \leq C \sum_{u=0}^{1}\left(\sum_{j \in \mathbb{Z}}\left|T_{\Omega ; b, u}^{*}\left(\Delta_{k-j ; b, 1-u}^{2} f\right)(x)\right|^{2}\right)^{\frac{1}{2}}
$$

where

$$
T_{\Omega ; b, u}^{*} f(x)=\sup _{r>0} \frac{1}{r^{n}} \int_{|x-y|<r}|f(y)||b(x)-b(y)|^{u}|\Omega(x-y)| d y .
$$

Since for $1<p<\infty$ and $u \in\{0,1\}$,

$$
\left\|\left(\sum_{j \in \mathbb{Z}}\left|T_{\Omega ; b, u}^{*} f_{j}\right|^{2}\right)^{1 / 2}\right\|_{L^{p}} \leq C\|b\|_{*}^{u}\|\Omega\|_{L \log ^{+} L\left(\mathbf{S}^{n-1}\right)}\left\|\left(\sum_{j \in \mathbb{Z}}\left|f_{j}\right|^{2}\right)^{1 / 2}\right\|_{L^{p}}
$$

which were established in [21] and [9]. Then by (3.26) and Lemma 3.4, we have for $1<p<\infty$,

$$
\left\|S_{2, k}\left(\mathcal{T}_{b} f\right)\right\|_{L^{p}} \leq C\|b\|_{*}\|\Omega\|_{L \log ^{+} L\left(\mathbf{S}^{n-1}\right)}\|f\|_{L^{p}} .
$$

This gives (3.15). Therefore, we complete the proof Proposition 3.6.

\section{Proof of Theorem 1.7}

We write

$$
\begin{aligned}
\Omega\left(x^{\prime}\right) & =\left[\Omega\left(x^{\prime}\right)-\frac{1}{\omega_{n-1}} \int_{\mathbf{S}^{n-1}} \Omega\left(y^{\prime}\right) d \sigma\left(y^{\prime}\right)\right]+\frac{1}{\omega_{n-1}} \int_{\mathbf{S}^{n-1}} \Omega\left(y^{\prime}\right) d \sigma\left(y^{\prime}\right) \\
& :=\Omega_{0}\left(x^{\prime}\right)+C(\Omega, n),
\end{aligned}
$$

where $\omega_{n-1}$ denotes the area of $\mathbf{S}^{n-1}$. Thus,

$$
\begin{aligned}
M_{\Omega, t ; b} f(x) & =\frac{1}{t^{n}} \int_{|x-y|<t} \Omega_{0}(x-y)(b(x)-b(y)) f(y) d y+C(\Omega, n) \frac{1}{t^{n}} \int_{|x-y|<t}(b(x)-b(y)) f(y) d y \\
& :=M_{\Omega_{0}, t ; b} f(x)+C(\Omega, n) M_{t ; b} f(x)
\end{aligned}
$$


where $\Omega_{0}$ satisfies the cancelation condition (1.11). Denote the operator family $\left\{M_{\Omega_{0}, t ; b}\right\}_{t>0}$ by $\mathcal{M}_{\Omega_{0} ; b}$ and $\left\{M_{t ; b}\right\}_{t>0}$ by $\mathcal{M}_{b}$. By Corollary 1.5, we get for $1<p<\infty$,

$$
\left\|V_{\rho} \mathcal{M}_{b}(f)\right\|_{L^{p}} \leq C\|b\|_{*}\|f\|_{L^{p}} .
$$

To prove Theorem 1.7, it suffices to show

$$
\left\|V_{\rho} \mathcal{M}_{\Omega_{0} ; b}(f)\right\|_{L^{p}} \leq C\|b\|_{*}\|\Omega\|_{L \log ^{+} L\left(S^{n-1}\right)}\|f\|_{L^{p}} .
$$

Similarly, the proof of (4.1) is reduced to prove

$$
\left\|V_{\rho}\left(\left\{M_{\Omega_{0}, 2^{k} ; b} f\right\}_{k \in \mathbb{Z}}\right)\right\|_{L^{p}} \leq C\|b\|_{*}\|\Omega\|_{L \log ^{+} L\left(S^{n-1}\right)}\|f\|_{L^{p}}
$$

and

$$
\left\|S_{2}\left(\mathcal{M}_{\Omega_{0} ; b} f\right)\right\|_{L^{p}} \leq C\|b\|_{*}\|\Omega\|_{L\left(\log ^{+} L\right)^{2}\left(S^{n-1}\right)}\|f\|_{L^{p}}
$$

For (4.2), the pointwise domination

$$
V_{\rho}\left(\left\{M_{\Omega_{0}, 2^{k} ; b} f\right\}_{k \in \mathbb{Z}}\right) \leq\left(\sum_{k}\left|M_{\Omega_{0}, 2^{k} ; b} f\right|^{2}\right)^{1 / 2}
$$

for $1<p<\infty$, which is a known result in [30].

For (4.3), observe that $V_{2, j}\left(\mathcal{M}_{\Omega_{0} ; b} f\right)$ is just the strong 2-variation function of the family $\left\{M_{\Omega_{0}, 2^{j} t ; b} f\right\}_{t \in[1,2)}$, hence

$$
S_{2}\left(\mathcal{M}_{\Omega_{0} ; b} f\right)(x)=\left(\sum_{j \in \mathbb{Z}}\left|V_{2, j}\left(\mathcal{M}_{\Omega_{0} ; b} f\right)(x)\right|^{2}\right)^{\frac{1}{2}} .
$$

Similar to the proof of (3.2), we get that for $1<p<\infty$,

$$
\left\|S_{2}\left(\mathcal{M}_{\Omega_{0} ; b} f\right)\right\|_{L^{p}} \leq C\|b\|_{*}\left\|\Omega_{0}\right\|_{L\left(\log ^{+} L\right)^{2}\left(S^{n-1}\right)}\|f\|_{L^{p}} \leq C\|b\|_{*}\|\Omega\|_{L\left(\log ^{+} L\right)^{2}\left(S^{n-1}\right)}\|f\|_{L^{p}} .
$$

Therefore, (4.3) is proved.

\section{References}

[1] J. Alvarez, R. Bagby, D. Kurtz and C. Pérez, Weighted estimates for commutators of linear operators, Studia Math. 104 (1993), 195-209.

[2] M. Bramanti and M. Cerutti, Commutators of singular integrals on homogeneous spaces, Boll. Un. Mat. Ital. B(7), 10 (1996), 843-883.

[3] J. M. Bony, Calcul symbolique et propagation des singularites pour les equations aux derivees partielles non lineaires, Ann. Sci. Ecole Norm. Sup.(4), 14 (1981), 209-246.

[4] J. Bourgain, Pointwise ergodic theorems for arithmetic sets, Inst. Hautes Études SCI, Publ. Math. 69 (1989), 5-41. 
[5] J. Campbell, R. Jones, K. Reinhold and M. Wierdl, Oscillation and variation for the Hilbert transform, Duke Math. J. 105 (2000), 59-83.

[6] J. Campbell, R. Jones, K. Reinhold and M. Wierdl, Oscillation and variation for singular integrals in higher dimensions, Trans. Amer. Math. Soc. 355 (2002), 2115-2137.

[7] A. P. Calderón and A. Zygmund, On singular integrals, Amer. J. Math., 78 (1956), 289-309.

[8] A. P. Calderón, On commutators of singular integrals, Studia Math. 53 (1975), 139-174.

[9] Y. Chen and Y. Ding, $L^{2}$ boundedness for commutator of rough singular integral with variable kernel, Rev. Mat. Iberoam. 24 (2008), 531-547.

[10] Y. Chen, Y. Ding, G. Hong and H. Liu, Weighted jump and variational inequalities for rough operators, submitted.

[11] Y. Chen, Y. Ding, G. Hong and J. Xiao, Some jump and variational inequalities for the Calderón commutators and related operators, submitted.

[12] Y. Chen and Y. Ding, $L^{p}$ bounds for the commutators of singular integrals and Maximal singular integrals with rough kernels, Tran. Amer. Math. Soc. 367 (2015), 1585-1608.

[13] F. Chiarenza, M. Frasca and P. Longo, Interior $W^{2, p}$ estimates for nondivergence elliptic equations with discontinuous coefficiens, Ric. Math. XL (1991), 149-168.

[14] F. Chiarenza, M. Frasca and P. Longo, $W^{2, p}$-solvability of the Dirichlet problem for nondivergence elliptic equations with VMO coefficients, Trans. Amer. Math. Soc. 334 (1993) 841-853.

[15] D. Chung, C. Pereyra and C. Pérez, Sharp bounds for general commutators on weighted Lebesgue spaces, Trans. Amer. Math. Soc. 364 (2012), 1163-1177.

[16] R. Coifman, P. Lions, Y. Meyer and S. Semmes, Compensated compactness and Hardy spaces, J. Math. Pures Appl. 72 (1993), 247-286.

[17] R. Coifman, R. Rochberg and G. Weiss, Factorization theorems for Hardy spaces in several variables, Ann. Math. 103 (1976), 611-635.

[18] Y. Ding, G. Hong and H. Liu, Jump and variational inequalities for rough operators, J. Four. Anal. Appl. (2016), DOI 10.1007/s00041-016-9484-8.

[19] G. Di Fazio and M. A. Ragusa, Interior estimates in morrey spaces for strong solutions to nondivergence form equations with discontinuous coefficients, J. Funct. Anal. 112 (1993), 241-256.

[20] Y. Do, C. Muscalu and C. Thiele, Variational estimates for paraproducts, Rev. Mat. Iberoam. 28 (2012), 857-878.

[21] J. Duoandikoetxea and J. Rubio de Francia, Maximal and singular integral operators via Fourier transform estimates, Invent. Math. 84 (1986), 541-561. 
[22] J. Garcia-Cuerva, E. Harboure, C. Segovia and J. L. Torre, Weighted norm inequality for commutators of strongly singular integrals, Indiana Univ. Math J. 40 (1991), 1397-1420.

[23] T. Gillespie and J. Torrea, Dimension free estimates for the oscillation of Riesz transforms, Israel J. Math. 141 (2004), 125-144.

[24] L. Grafakos, Classical and Modern Fourier Analysis, Pearson Education, Inc. Upper Saddle River, New Jersey, 2004.

[25] L. Greco and T. Iwaniec, New inequalities for the Jacobian, Ann. Inst. H. Poincaré 11 (1994), 17-35.

[26] G. Hong, The behaviour of square functions from ergodic theory in $L^{\infty}$, Proc. Amer. Math. Soc. 143(11) (2015), 4797-4802.

[27] G. Hong and T. Ma, Vector-valued q-variation for differential operators and semigroups I, Math. Z. DOI 10.1007/s00209-016-1756-0.

[28] G. Hong, T. Ma, Vector-valued q-variation for ergodic averages and analytics semigroups, J. Math. Anal. Appl. 437(2016), 1084-1100.

[29] G. Hu, $L^{p}\left(\mathbb{R}^{n}\right)$ boundedness for the commutator of a homogeneous singular integral operator, Studia Math. 154 (2003), 13-27.

[30] G. Hu and D. Yan, On the commutator of the Marcinkiewicz integral, J. Math. Anal. Appl. 283 (2003), 351-361.

[31] T. Hytönen, M. Lacey and I. Parissis, A variation norm Carleson theorem for vector-valued Walsh-Fourier series, Rev. Mat. Iberoam. 30 (2014), 979-1014.

[32] R. Jones, R. Kaufman, J. Rosenblatt and M. Wierdl, Oscillation in ergodic theory, Ergodic Theory Dynam. Systems. 18 (1998), 889-935.

[33] R. Jones, J. Rosenblatt and M. Wierdl, Oscillation inequalities for rectangles, Proc. Amer. Math. Soc. 129 (2000), 1349-1358.

[34] R. Jones, J. Rosenblatt and M. Wierdl, Oscillation in ergodic theory: Higher dimensional results, Israel J. Math. 135 (2003), 1-27.

[35] R. Jones, A. Seeger and J. Wright, Strong variational and jump inequalities in harmonic analysis, Trans. Amer. Math. Soc. 360 (2008), 6711-6742.

[36] B. Krause, Polynomial Ergodic Averages converge rapidly: variations on a theorem of Bourgain, arXiv: 1402.1803.

[37] B. Krause and P. Zorin-Kranich, Weighted and vector-valued variational estimates for ergodic averages, arXiv: 1409.7120 .

[38] C. Le Merdy and Q. Xu, Strong q-variation inequalities for analytic semigroups, Ann. Inst. Fourier. 62 (2012), 2069-2097. 
[39] D. Lépingle, La variation d'ordre p des semi-martingales, Z. Wahrsch. Verw. Gebiete. 36 (1976), 295-316.

[40] F. Liu and $\mathrm{H}$. Wu, A criterion on oscillation and variation for the commutators of singular integral operators, Forum Math. 27 (2015), 77 -97.

[41] T. Ma, J. Torrea and Q. Xu, Weighted variation inequalities for Differential operators and Singular integrals, J. Funct. Anal. 268(2015), 376-416.

[42] T. Ma, J. Torrea and Q. Xu, Weighted variation inequalities for Differential operators and Singular integrals in higher dimensions. arXiv:1511.05129, 2015.

[43] A. Mas and X. Tolsa, Variation for the Riesz transform and uniform rectifiability, J. Eur. Math. Soc. 16 (2014), 2267-321.

[44] M. Mirek, B. Trojan, Discrete maximal functions in higher dimensions and applications to ergodic theory, arXiv: 1405.5566.

[45] M. Mirek, E. M. Stein and B. Trojan, $\ell_{p}\left(Z^{d}\right)$-estimates for discrete operators of Radon type: Variational estimates, arXiv: 1512.07523.

[46] M. Mirek, B. Trojan and P. Zorin-Kranich, Variational estimates for averages and truncated singular integrals along the prime numbers, arXiv: 1410.3255.

[47] R. Oberlin, A. Seeger, T. Tao, C. Thiele and J. Wright, A variation norm Carleson theorem, J. Eur. Math. Soc. 14 (2012), 421-464.

[48] G. Pisier and Q. Xu, The strong p-variation of martingales and orthogonal series, Probab. Theory Related Fields. 77 (1988), 497-514.

[49] J. Qian, The p-variation of partial sum processes and the empirical process, Ann. Prob. 77 (1998), 1370-1383.

[50] R. Rochberg and G. Weiss, Derivatives of analytic families of Banach spaces, Ann. Math. 118 (1983), 315-347.

[51] M. Taylor, Commutator estimates for Hölder continuous and bmo-Sobolev multipliers, Proc. Amer. Math. Soc. 143 (2015), 5265-5274.

[52] P. Zorin-Kranich, Variation estimates for averages along primes and polynomials, arXiv: 1403.4085 . 